\title{
RNA-binding protein IGF2BP3 targeting of oncogenic transcripts promotes hematopoietic progenitor proliferation
}

\author{
Jayanth Kumar Palanichamy, ${ }^{1}$ Tiffany M. Tran, ${ }^{1,2}$ Jonathan M. Howard, ${ }^{3}$ Jorge R. Contreras, ${ }^{1}$ Thilini R. Fernando, ${ }^{1}$ \\ Timothy Sterne-Weiler, ${ }^{3}$ Sol Katzman, ${ }^{3}$ Masoud Toloue, ${ }^{4}$ Weihong Yan, ${ }^{5}$ Giuseppe Basso, ${ }^{6}$ Martina Pigazzi, ${ }^{6}$ \\ Jeremy R. Sanford, ${ }^{3}$ and Dinesh S. Rao ${ }^{1,2,7,8}$
}

'Department of Pathology and Laboratory Medicine, David Geffen School of Medicine at UCLA, Los Angeles, California, USA. ${ }^{2}$ Department of Molecular, Cellular and Integrative Physiology, UCLA, Los Angeles, California, USA. ${ }^{3}$ Department of Molecular, Cellular and Developmental Biology, UCSC, Santa Cruz, California, USA. " $B$ Bioo Scientific Corporation, Austin, Texas, USA. ${ }^{5}$ Department of Chemistry and Biochemistry, UCLA, Los Angeles, California, USA. ${ }^{6}$ Department of Women's and Children's Health SDB, University of Padova, Padova, Italy. ${ }^{7}$ Jonsson Comprehensive Cancer Center (JCCC) and ${ }^{8}$ Broad Stem Cell Research Center, UCLA, Los Angeles, California, USA.

\begin{abstract}
Posttranscriptional control of gene expression is important for defining both normal and pathological cellular phenotypes. In vitro, RNA-binding proteins (RBPs) have recently been shown to play important roles in posttranscriptional regulation; however, the contribution of RBPs to cell specification is not well understood. Here, we determined that the RBP insulin-like growth factor 2 mRNA-binding protein 3 (IGF2BP3) is specifically overexpressed in mixed lineage leukemia-rearranged (MLLrearranged) B-acute lymphoblastic leukemia (B-ALL), which constitutes a subtype of this malignancy associated with poor prognosis and high risk of relapse. ICF2BP3 was required for the survival of B-ALL cell lines, as knockdown led to decreased proliferation and increased apoptosis. Enforced expression of ICF2BP3 provided murine BM cells with a strong survival advantage, led to proliferation of hematopoietic stem and progenitor cells, and skewed hematopoietic development to the B cell/myeloid lineage. Cross-link immunoprecipitation and high throughput sequencing uncovered the ICF2BP3-regulated transcriptome, which includes oncogenes $M Y C$ and $C D K 6$ as direct targets. ICF2BP3 regulated transcripts via targeting elements within $3^{\prime}$ untranslated regions ( $3^{\prime} U T R$ ), and enforced IGF2BP3 expression in mice resulted in enhanced expression of Myc and Cdk6 in BM. Together, our data suggest that ICF2BP3-mediated targeting of oncogenic transcripts may represent a critical pathogenetic mechanism in MLL-rearranged B-ALL and support IGF2BP3 and its cognate RNA-binding partners as potential therapeutic targets in this disease.
\end{abstract}

\section{Introduction}

Oncogenesis in early B cell progenitors results in B cell acute lymphoblastic leukemia (B-ALL), the most prevalent hematological neoplasm in children and young adults (1). The majority of B-ALL cases exhibit genetic alterations, including recurring chromosomal rearrangements, which contribute to the heterogeneity of the observed clinical behavior (2). Specifically, B-ALL with chromosomal rearrangements of the mixed lineage leukemia $(M L L)$ gene accounts for $5 \%-6 \%$ of all B-ALL cases and is associated with poor prognosis and risk of early relapse after treatment (3). $M L L$, which encodes a H3K4 methyltransferase, plays a critical role in the transcriptional dysregulation that occurs during leukemogenesis (3, 4). Previously demonstrated targets of MLL include genes critical in cell survival and proliferation, such as BCL2, MYC, and CDK6 (5-7). Additionally, MLL is known to regulate hematopoiesis, and its expression correlates with the maintenance of hematopoietic

Authorship note: J. Kumar Palanichamy, T.M. Tran, and J.M. Howard contributed equally to this work.

Conflict of interest: The authors have declared that no conflict of interest exists. Submitted: November 17, 2014; Accepted: January 26, 2016.

Reference information: / Clin Invest. 2016;126(4):1495-1511. doi:10.1172/JCI80046. stem cell (HSC) self-renewal and differentiation $(8,9)$. In line with such a role in normal HSC function, MLL fusion proteins induce HOXA9 and MEIS1, generating leukemia that displays stem celllike properties (10-12). These findings demonstrate an intimate connection between the dysregulation of gene expression and malignant transformation, and they highlight the importance of investigating key players in the regulation of gene expression.

Simplistically, gene expression may be regulated at the transcriptional and posttranscriptional levels. Recent work has revealed the complexity of the latter mechanism, which not only includes sequences intrinsic to the regulated mRNA but also other factors such as miRs, RNA-binding proteins (RBPs), and noncoding RNA (13). A complex interplay between the protein coding mRNA and the $3^{\prime}$ untranslated region ( $3^{\prime} \mathrm{UTR}$ ) targeting miRs and RBPs has been reported (14). However, the role of gene expression regulation by RBPs in the malignant transformation of B cells is not understood. In an effort to identify critical RBP-mediated regulation in B-ALL, we began by examining a high throughput dataset generated in our laboratory, identifying the insulin-like growth factor 2 mRNA-binding protein 3 (IGF2BP3) as one of the top dysregulated genes in MLL-translocated B-ALL. IGF2BP3 belongs to a family of mRNA-binding proteins that consists of 3 structurally and 
functionally related paralogs (IGF2BP1, IGF2BP2, and IGF2BP3) that influence the cytoplasmic fate of mRNAs through localization, stability, and translation $(15,16)$. IGF2BP3 is an oncofetal protein with high expression during embryogenesis, low expression in adult tissues, and reexpression in malignant tissues. In epithelial cancer, IGF2BP3 expression is associated with a range of neoplastic phenotypes (17-20). However, many of these studies have been largely correlative, and a bona fide functional role of IGF2BP3, or any RBP, in B cell oncogenesis has not been established.

In this study, we sought to delineate the function of IGF2BP3 in B cell leukemogenesis. We overexpressed IGF2BP3 in the BM of lethally irradiated mice and found that it plays a critical role in the proliferation of hematopoietic stem and progenitor cells, recapitulating some features of MLL-rearranged B-ALL. IGF2BP3 provided BM progenitors with a competitive survival advantage and increased their proliferation. We also found that IGF2BP3 was essential for the survival of B-ALL cell lines. We used individual nucleotide resolution cross-linking immunoprecipitation (iCLIP) to capture the in situ specificity of protein-RNA interactions and to reveal the positional context of protein binding sites across the transcriptome. In total, we identified IGF2BP3 binding sites in several hundred transcripts in $2 \mathrm{~B}-\mathrm{ALL}$ cell lines. IGF2BP3 cross-linking sites are strongly enriched in the $3^{\prime} \mathrm{UTR}$ s of target transcripts. Of the many IGF2BP3 target transcripts, we demonstrated IGF2BP3-mediated enhancement of the expression of oncogenic targets CDK6 and MYC in B-ALL cells and hematopoietic progenitor cells in vivo. Deletion of the RNA-binding domains of IGF2BP3 abrogated target mRNA binding as well as the hematopoietic stem and progenitor expansion. Together, our studies suggest that IGF2BP3-mediated upregulation of oncogenic targets represents a key pathogenetic mechanism operant in MLL-rearranged B-ALL.

\section{Results}

IGF2BP3 is differentially expressed in MLL-rearranged B-ALL. We have previously described a microarray experiment performed on patient B-ALL samples (21). Following correction for multiple-hypotheses testing, we performed unsupervised hierarchical clustering with significantly differentially expressed protein-coding genes (adjusted $P \leq 0.01$ ). This generated a list of RBPs differentially expressed between the 3 cytogenetic subtypes of B-ALL used in our microarray experiments (ETV-RUNX1, E2A-PBX, and MLL-rearranged). In the list of RBPs whose expression was highest in MLL-rearranged leukemia, IGF2BP3 was among the top candidates (Figure 1A). MLL-rearranged leukemias show a stem-cell signature with high expression of stemness-associated genes like HOXA9, MEIS1, and CD44 $(11,22)$. Concordant with this, we observed that HOXA9, MEIS1A, CDK6, and MYC - putative targets of the oncogenic MLL fusion protein - were significantly overexpressed in the MLL-rearranged group when compared with the other 2 subsets (Supplemental Figure 1, A-D). By performing quantitative PCR (qPCR) on a large cohort of B-ALL patient-derived BMs, we confirmed that IGF2BP3 and CD44 were highly expressed in the MLL group (total $n=134$ ) (Figure $1, \mathrm{~B}$ and C). Additionally, IGF2BP3 expression was significantly higher in all B-ALL samples when compared with CD19+ $\mathrm{B}$ cells isolated from healthy donors (Figure 1B). To examine the dependence of IGF2BP3 on MLL-mediated effects on gene expression, we utilized I-BET151, a bromodomain and extra terminal (BET) domain inhibitor that has recently been shown to inhibit MLLdependent gene expression (23). Treatment of RS4;11 - an MLLAF4-expressing human B-ALL cell line - with I-BET151 caused a dose-dependent decrease in the expression of $M Y C, C D K 6$, and IGF2BP3 (Figure 1D). It also caused cell cycle arrest in the G1-S phase (Figure 1, E and F). These experiments confirm the overexpression of IGF2BP3 in B-ALL, with the highest expression seen in MLL-rearranged B-ALL. In line with IGF2BP3 being downstream of MLL fusion proteins, a fall in IGF2BP3 mRNA levels, along with a fall in other MLL-AF4 target levels, is seen after BET inhibition.

IGF2BP3 loss of function causes apoptosis in B-ALL cells. Given the oncogenic expression pattern of IG2BP3 in human B-ALL, we proceeded to examine its expression in 4 different B-ALL cell lines, including 697 (E2A-PBX translocated), RS4;11, REH (ETV-RUNX1 translocated), and NALM6 (Figure 2A). To examine the effects of IGF2BP3 knockdown, we used a lentiviral vector expressing 2 different miR formatted siRNA sequences to transduce RS4;11 cells (Figure 2B). Both siRNAs caused decreased IGF2BP3 expression by qPCR (Figure 2C). Propidium iodide staining showed an increase in the apoptotic sub-G1 fraction, and 3-(4,5-dimethylthiazol-2-yl)-5-(3-carboxymethoxyphenyl)-2-(4sulfophenyl)-2H-tetrazolium (MTS) assay showed a significant reduction in cell proliferation with IGF2BP3 knockdown, confirming the dependence of B-ALL cell lines on IGF2BP3 for survival (Figure 2, D and E). Deletion of the IGF2BP3 locus using the CRISPR-Cas9 system was also undertaken in the RS4;11 cell line. We utilized the LentiCRISPR system (24) with 2 different guide strands, Cr1 and Cr2, to target the IGF2BP3 locus for deletion. CRISPR-mediated deletion was confirmed by a T7 endonuclease assay (Supplemental Figure 2, D and E) with complete abrogation of IGF2BP3 protein with the guide RNA Cr2, whereas residual protein was detected with $\mathrm{Cr} 1$ (Figure $2 \mathrm{~F}$ ). Cr2-mediated deletion resulted in reduced cell proliferation by MTS assay, increased sub-G1 staining, and increased annexin V positivity (Figure 2, G-I). To confirm these findings, we also targeted IGF2BP3 for knockdown in NALM6 cells using a lentiviral siRNA expression system (Supplemental Figure 2A). Reduced IGF2BP3 mRNA levels were observed with both siRNAs, with si2 giving a stronger reduction in cell proliferation, as seen by the MTS assay (Supplemental Figure $2, \mathrm{~B}$ and $\mathrm{C})$. Together, these findings highlight the importance of IGF2BP3 in maintaining cell survival and proliferation in B-ALL.

Enforced expression of IGF2BP3 leads to high levels of engraftment and increased leukocytes. To directly assess the role of IGF2BP3 in the hematopoietic system, we undertook an in vivo experiment to examine the effects of enforced expression. We initially cloned the human or mouse coding sequence of IGF2BP3 into MIG, a murine stem cell virus-based (MSCV-based) retroviral vector (Figure 3A), and confirmed the functionality of the vector in expressing both IGF2BP3 and the GFP marker (Figure 3, B-D, and data not shown). A peripheral bleed of these mice at 4 weeks showed a significant increase in $\mathrm{GFP}^{+}$cells that was sustained over time in mice with enforced expression of human and mouse IGF2BP3, as measured by the congenic CD 45.2 versus CD45.1 FACS markers (Figure 3, E and $\mathrm{F}$ ). Moreover, significantly increased $\mathrm{GFP}^{+}$leukocyte cells were found, confirming increased hematopoietic output attributable to IGF2BP3 expression (Figure 3G). These changes were restricted to 


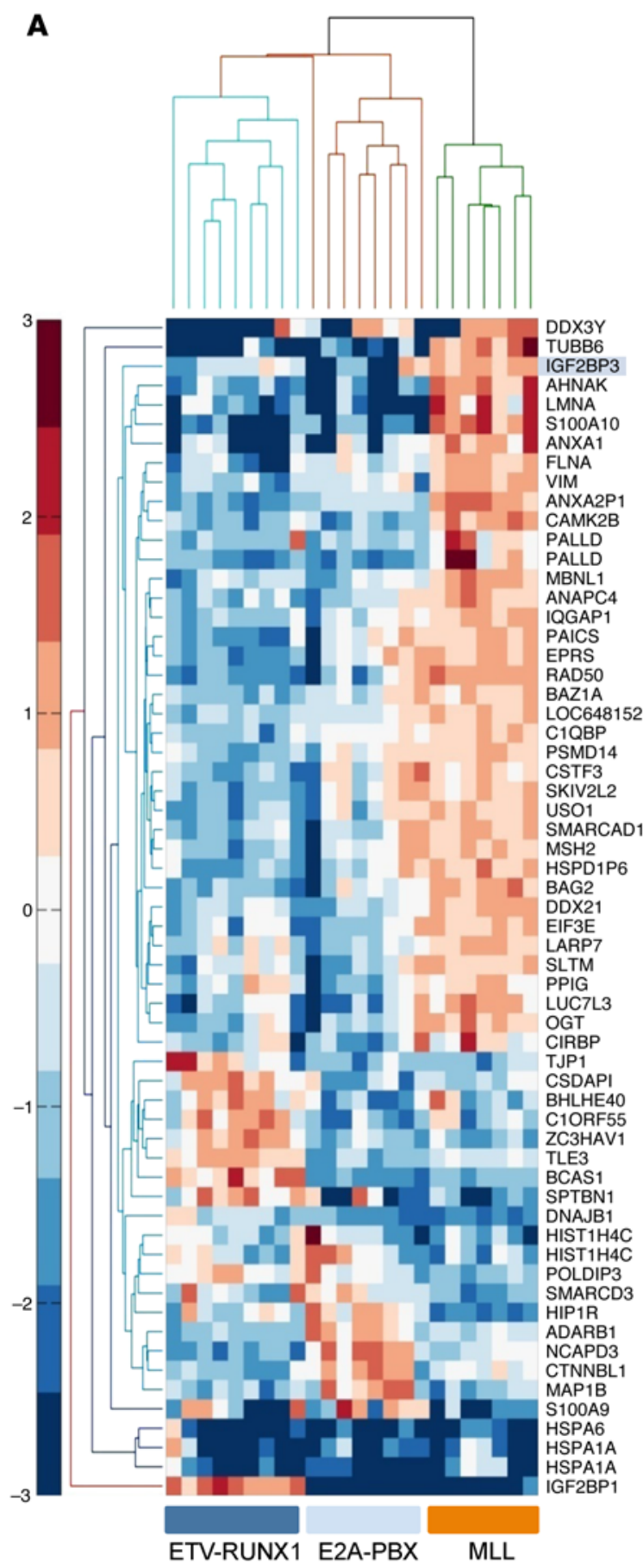

Figure 1. IGF2BP3 is overexpressed in MLL-translocated B-ALL. (A) Heatmap from the microarray data showing differentially expressed RBPs between B-ALL. IGF2BP3 is highly expressed in MLL-rearranged B-ALL. (B and C) QPCR-based confirmation of overexpression of ICF2BP3 (B) and its previously defined target, $C D 44$ (C), in MLL-rearranged B-ALL (total $n=134$; one-way ANOVA followed by Bonferroni's multiple comparisons test; ${ }^{* *} P<0.01,{ }^{* * *} P<0.001$, ${ }^{* * * *} P<0.0001$ ). (D-F) Treatment of RS4;11 cell line with increasing doses of I-BET151. (D) qPCR of MYC, CDK6, and IGF2BP3 levels in RS4;11 cells shows a significant decrease in all 3 mRNA levels ( $t$ test $M Y C, P=0.04, P=0.06$; IGF2BP3, $P<0.0001, P=0.1 ; C D K 6, P=0.005$, $P=0.004 ; 1 \mu \mathrm{M}$ and $2 \mu \mathrm{M}$, respectively). (E and $\mathbf{F}$ ) Cell cycle analysis by propidium iodide staining after I-BET151 treatment of RS4;11 cells shows G1 arrest secondary to CDK6 inhibition. Experiments were conducted $3 \times$ for validation. qPCR assays were normalized to actin (B and $\mathbf{C}$ ) and RNA Pol II (D). Data represent mean $\pm S D$. See also Supplemental Figure 1 .
B

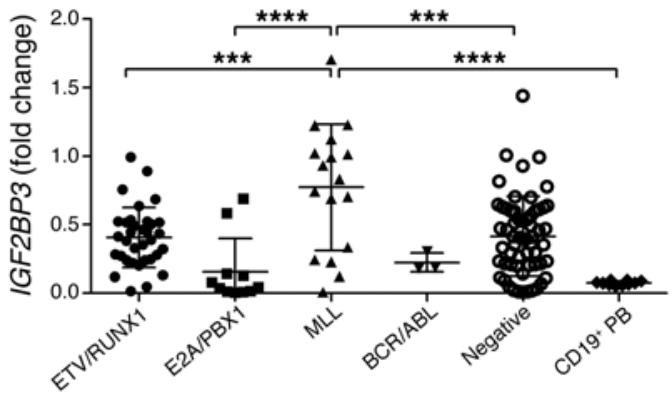

C

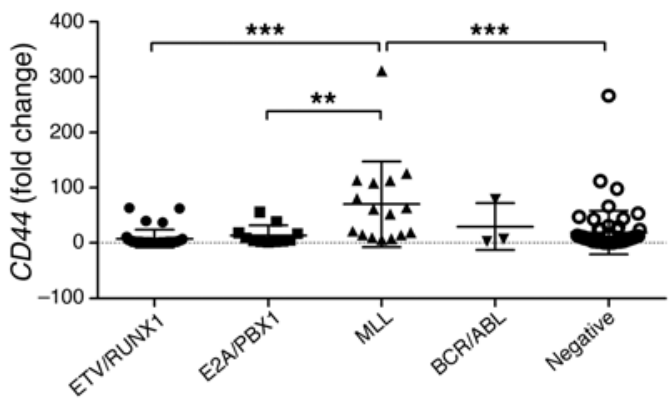

D

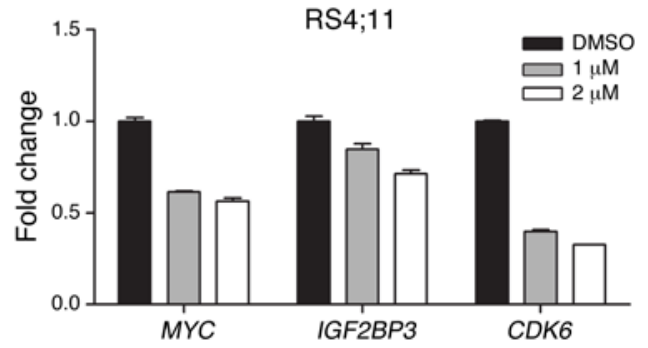

E

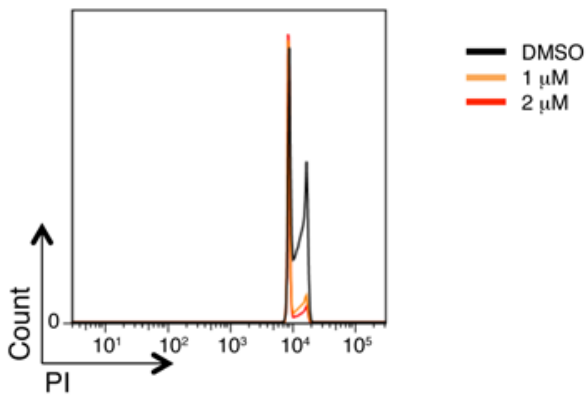

$\mathbf{F}$

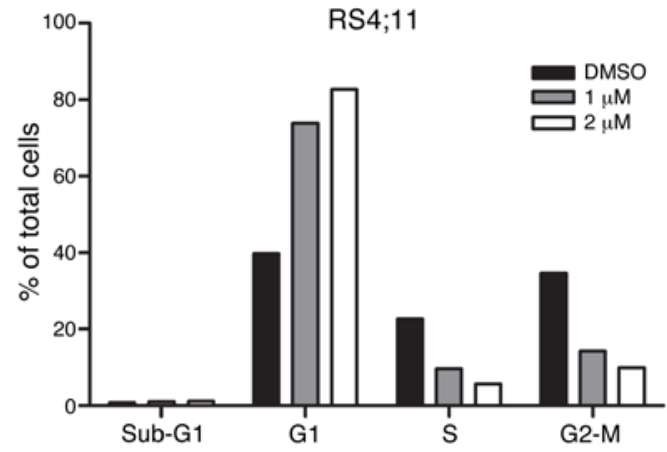


A
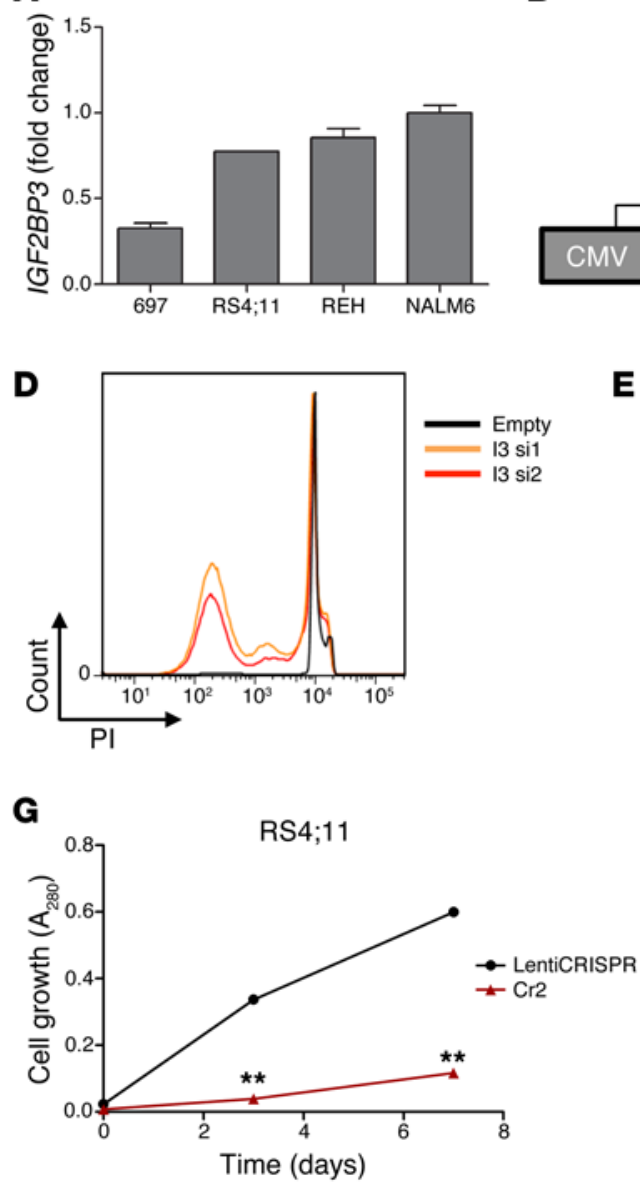

B

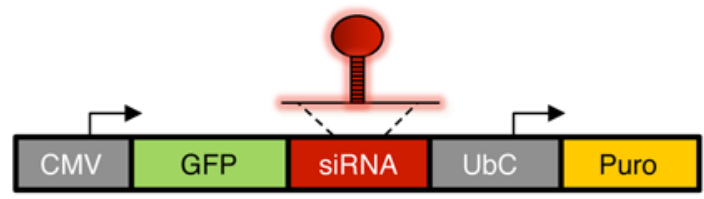

E

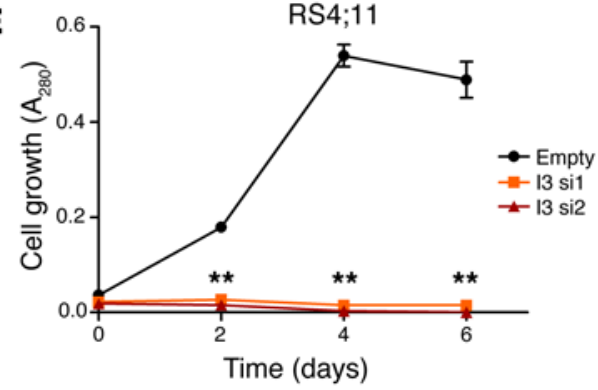

C

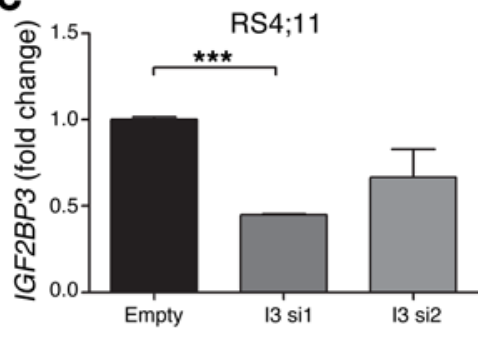

$\mathbf{F}$

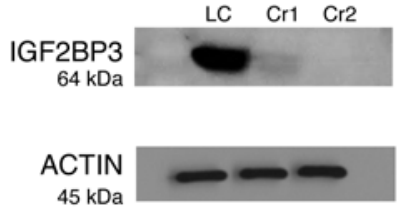

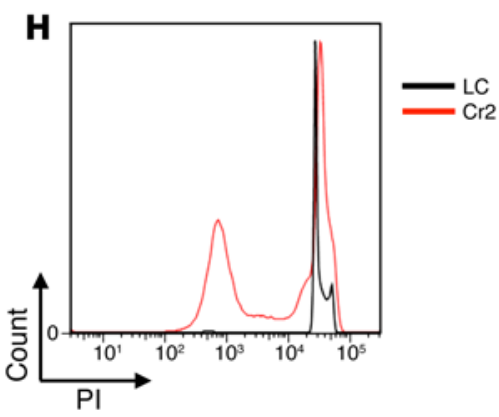

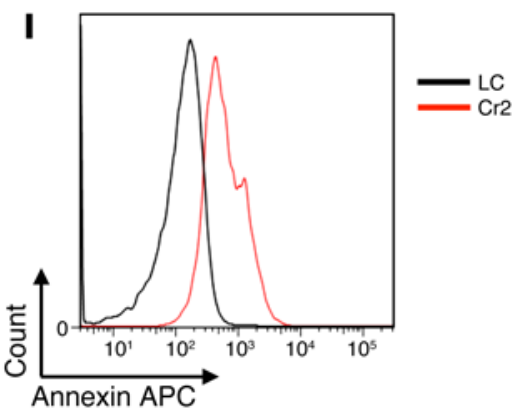

Figure 2. IGF2BP3 knockdown leads to disruptions of cell growth and increased apoptosis. (A) ICF2BP3 expression in human B-ALL cell lines. (B) Schematic of lentiviral vector used for ICF2BP3 knockdown. (C) IGF2BP3 knockdown, measured by qPCR shown in RS4;11 cell line ( $t$ test; *** $P=0.0005)$. (D) Cell cycle analysis with propidium iodide staining. (E) MTS assay showing significantly reduced cell proliferation with ICF2BP3 knockdown. (F) Western blot showing ICF2BP3 expression after CRISPR-Cas9-mediated targeting using the $\mathrm{Cr} 1 \mathrm{or} \mathrm{Cr} 2$ constructs. Cr1-mediated targeting results in some residual protein. $\beta$-Actin is used as a loading control. (C) MTS assay showing significantly reduced cell proliferation after $\mathrm{Cr} 2$ targeting ( $t$ test; ${ }^{* *} P \leq 0.01$ for all marked comparisons). (H) Cell cycle analysis by propidium iodide staining showing increased cell death (sub-G1 peak) in Cr2-expressing cells. (I) Increased annexin $V$ staining in $\mathrm{Cr}$ 2-targeted cells with IGF2BP3 KO. I3, IGF2BP3. Experiments were conducted $3 \times$ for validation. Data represent mean \pm SD. See also Supplemental Figure 2. UbC, ubiquitin C promoter; Puro, puromycin; LC, lentiCRISPR control.

the B cell and myeloid cell counts in the peripheral blood following complete engraftment (Figure 3, $\mathrm{H}$ and I). There was no difference in the number of $\mathrm{T}$ cells in the periphery (Figure 3J). Interestingly, the number of platelets and red blood cells were significantly lower with IGF2BP3-enforced expression (Figure 3, K and L). Together these findings suggest that IGF2BP3 promotes overall hematopoietic output from the BM and skews BM development toward the B cell/myeloid lineage and away from T-cells, erythroid cells, and megakaryocytes. These findings, notably the preferential increase in B cells and myeloid cells, are interesting in light of the fact that MLL rearrangements are found not only in B-ALL, but also in acute myeloid leukemia and mixed lineage acute leukemia, most commonly expressing both B cell and myeloid markers.

Enforced expression of IGF2BP3 leads to increased progenitors in the BM with higher rates of proliferation. To further characterize these hematopoietic changes, IGF2BP3-overexpressing mice were sacrificed and hematopoietic organs were collected for analysis at 6 months after transplant. The percentage of $\mathrm{GFP}^{+}$cells was sig- nificantly higher in the IGF2BP3-overexpressing BM, similar to the peripheral blood (Supplemental Figure 3, A and B). The overall proportion of myeloid and $\mathrm{B}$ cells in the $\mathrm{BM}$ were similar between control and IGF2BP3-expressing mice (Supplemental Figure 3, C and D). qPCR from the RNA collected from the mouse BM confirmed human and mouse IGF2BP3 overexpression (Supplemental Figure 3, E and F). These changes led us to query whether there were changes in hematopoietic progenitors in the BM. Indeed, enforced expression of IGF2BP3 led to an increase in the fraction of HSCs, lymphoid-primed multipotent progenitors (LMPPs), and common lymphoid progenitors (CLPs) (Figure 4, A-C). We followed the developmental pathway of B cells by following the schema created by Hardy et al. (25). Among the Hardy fractions, we observed a significant increase in the number of cells in fractions A and B with no significant differences observed in developmentally subsequent stages (Supplemental Figure 3, H, I, and K). Hence, overexpression of IGF2BP3 led to an increase in immature hematopoietic fractions starting at the level of the HSC and on to 
A

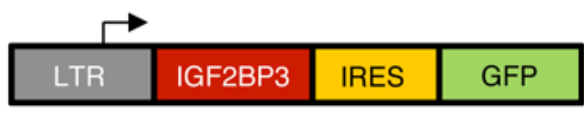

B (64 kDa) IGF2BP3 (45 kDa) ACTIN
70Z/3
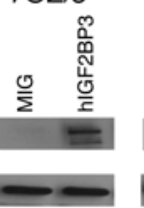

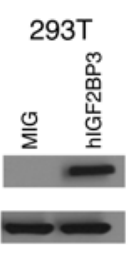

C
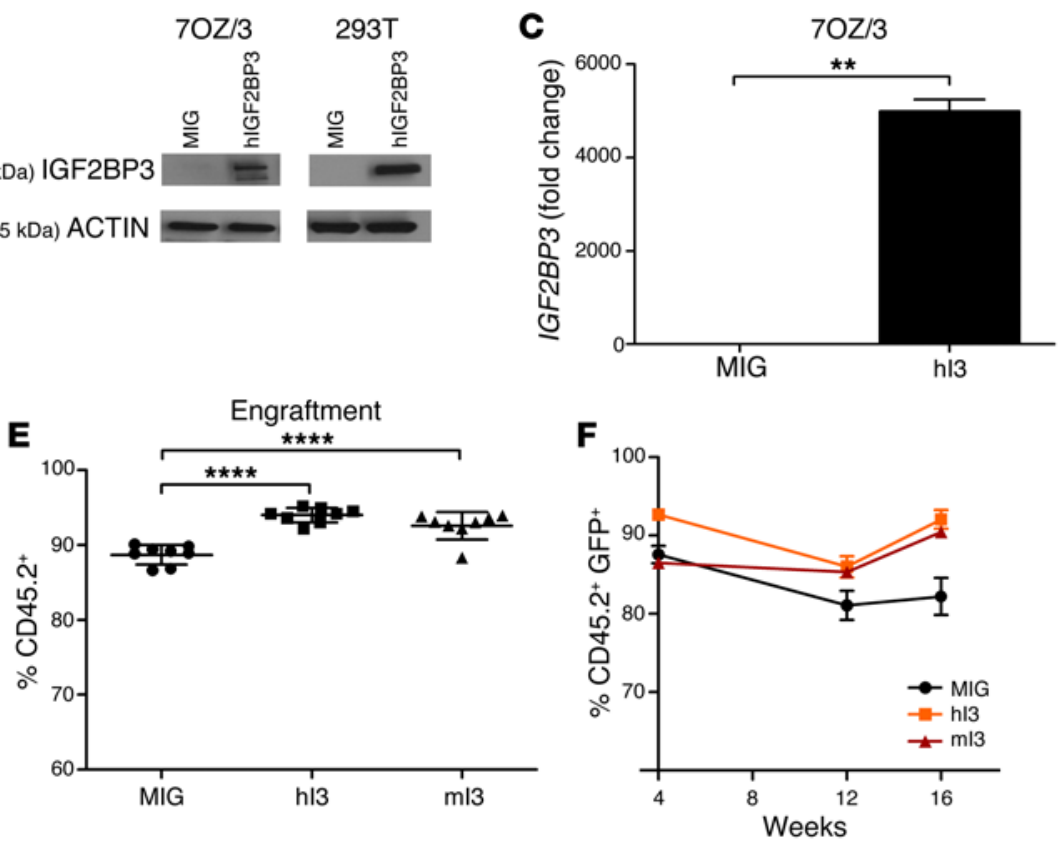

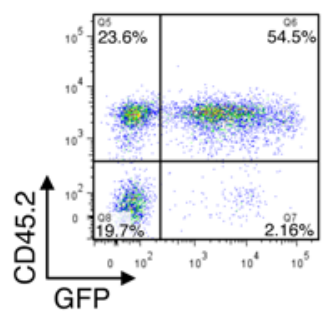

G
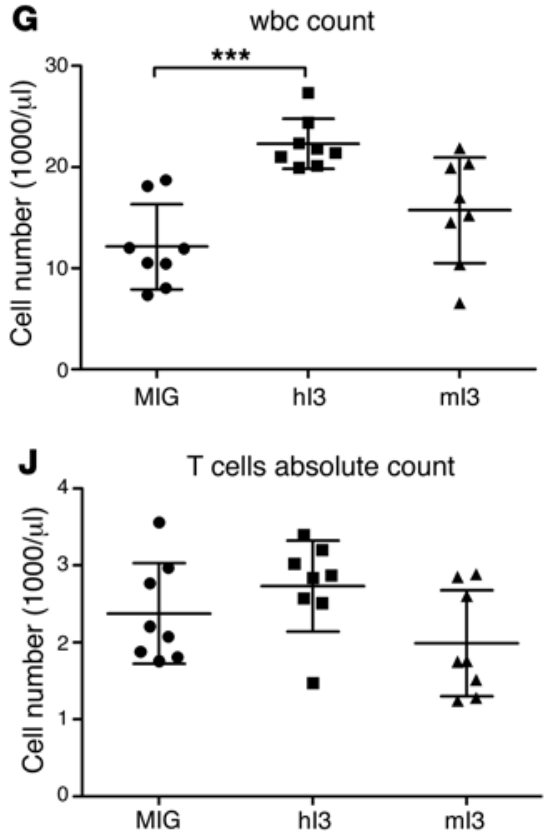

H

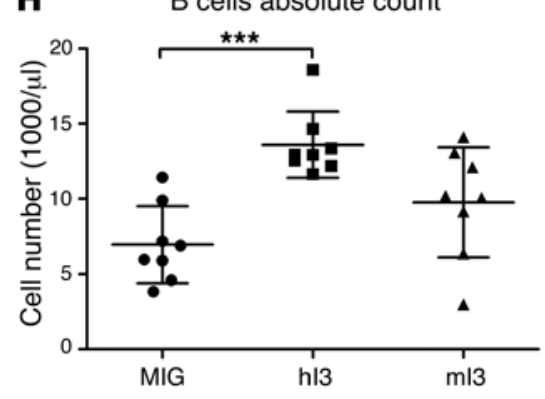

$\mathbf{K}$

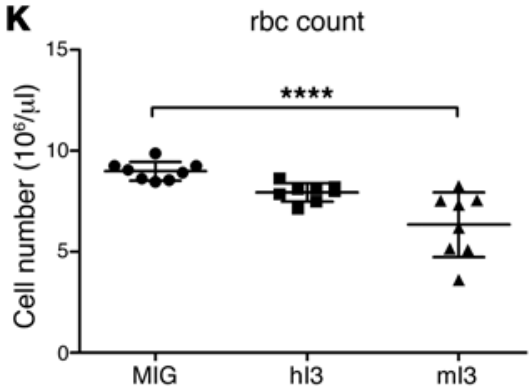

I

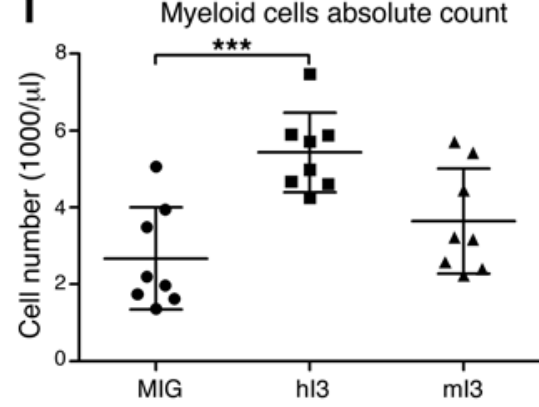

L

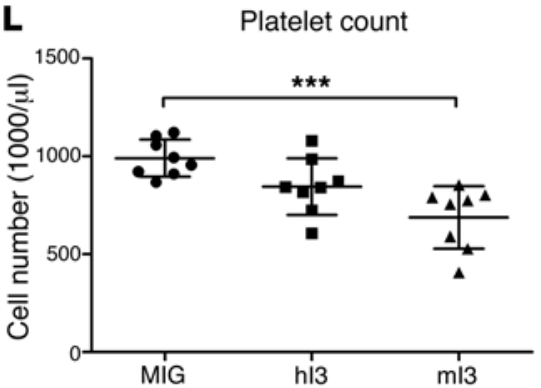

Figure 3. Enforced expression of IGF2BP3 leads to enhanced engraftment and skewing toward B cell/myeloid development. (A) Schematic of the bicistronic vector used for enforced expression of IGF2BP3. (B) Western blot showing overexpression of ICF2BP3 in the murine pre-B cell line, 70Z/3, and the human embryonic kidney cell line, 293T. (C) qPCR showing overexpression in 70Z/3 at the mRNA level ( $t$ test; ${ }^{* *} P=0.0013$ ). (D) FACS analysis of PB from mice 6 weeks after BMT showing successful engraftment and transduction (CFP+). (E) FACS of PB done at 4 weeks after BMT, showing CD45.2 and GFP positivity (one-way ANOVA followed by Bonferroni's test; ${ }^{* * *} P<0.0001$ ). (F) Quantitation of GFP expression in the PB between 4 and 16 weeks after transplant shows that the effect is marked and sustained. (G) PB leukocyte counts at 16 weeks show increased leukocytes (one-way ANOVA with Bonferroni's test; ${ }^{* * *} P<0.001$ ). ( $\mathbf{H}$ and $\left.\mathbf{I}\right)$ Significantly higher numbers of $B 220^{+}$cells $(\mathbf{H})$ and CD11 $\mathrm{b}^{+}$cells $(\mathbf{I})$ in PB (one-way ANOVA with Bonferroni's test; ${ }^{* * *} P<0.001$ ). (J) FACS-based enumeration of T cells shows no significant change in circulating T cells. (K and $\mathbf{L}$ ) Enumeration of RBCs and platelets by CBC show significant reductions (one-way ANOVA with Bonferroni's test; ${ }^{* *} P<0.001,{ }^{* * *} P<0.0001$ ). $n=8$ for all 3 groups. PB, peripheral blood; BMT, BM transplantation; hI3, human IGF2BP3; mI3, murine IGF2BP3; CBC, complete blood count; LTR, long terminal repeat; IRES, internal ribosome entry site. Three separate BMT experiments were completed for validation. Data represent mean \pm SD.

the pro-B cell stage. To analyze the proliferation rate of the various progenitor cells in the BM, we performed intracellular staining with Ki67 in conjunction with progenitor cell stains. Ki67 was significantly higher in $\mathrm{Lin}^{-} \mathrm{Scal}^{+} \mathrm{c}-\mathrm{Kit}^{+}$(LSK) population and the
LMPPs in IGF2BP3-overexpressing BM (Figure 4, D and E). The CLPs did not show a significant difference in Ki67 expression (Supplemental Figure 3, G and J). These findings imply an increase in the proliferation rate of the early progenitors (HSCs and LMPPs), 
secondary to increased IGF2BP3 expression. Presumably, this leads to an increase in their numbers and differentiation into more committed downstream progenitors (CLPs and Hardy fractions A and B). Hence, the enforced expression of IGF2BP3 causes a preferential increase in numbers and proliferation of early progenitor populations, leading to the observed B cell- and myeloid biased leukocytosis seen in the periphery.

IGF2BP3 increases the number of B cells in the thymus and myeloid cells in the spleen. The normal mouse thymus is composed mostly of $\mathrm{T}$ cell progenitors, but in many murine models of leukemia and lymphoma, it becomes enlarged and overrun by malignant leukocytes (26). On microscopic examination, IGF2BP3 caused thymic medullary expansion with infiltration by large cells. One of the thymi expressing human IGF2BP3 had complete ablation of the cortico-medullary junction (Figure 5A). Hence, IGF2BP3 expression may serve as a precursor to malignant transformation. We observed a significantly higher percentage of $\mathrm{GFP}^{+} \mathrm{B} 22 \mathrm{O}^{+}$ $\mathrm{B}$ cells in the thymus when human or mouse IGF2BP3 was overexpressed, with the effect being more pronounced with mouse IGF2BP3 (Figure 5, B and C). Mice with enforced expression of mouse IGF2BP3 also showed a substantial decrease in the number of $\mathrm{CD} 3 \varepsilon^{+} \mathrm{T}$ cells. There was no significant difference in the level of $\mathrm{GFP}^{+}$cells in these thymi, indicating a lineage-specific expansion of B cells (Supplemental Figure 4, G-I). Four of 8 of the thymi overexpressing human IGF2BP3 and $1 / 8$ of the thymi overexpressing mouse IGF2BP3 weighed over $50 \mathrm{mg}$, with no such increase in control mice (data not shown). Interestingly, the spleens were also enlarged following enforced expression of IGF2BP3. Differences in splenic weight were statistically significant for mice with overexpression of human IGF2BP3 with a trend noted for the mouse IGF2BP3 group (Supplemental Figure 4A). IGF2BP3 led to an increase in the number of myeloid cells in the spleen with a significant decrease in the number of $\mathrm{CD} 3 \varepsilon^{+} \mathrm{T}$ cells (Supplemental Figure 4, B-F). Overall, IGF2BP3 appears to tilt the hematopoietic developmental program toward the $\mathrm{B}$ cell and myeloid lineages. Hence, the changes seen in the BM - increased numbers and proliferation of B-lymphoid and myeloid progenitors - may result in alterations in hematopoietic homeostasis in the periphery.

IGF2BP3 provides hematopoietic progenitors with a survival advantage. To confirm that IGF2BP3 overexpression equipped the $\mathrm{BM}$ progenitors with an advantage while repopulating the irradiated host mouse BM, we performed a formal competitive repopulation transplant assay. Fifty percent of CD45.1 BM cells were mixed with $50 \%$ of MIG or IGF2BP3- or HOXA9-overexpressing CD45.2 BM cells and injected into lethally irradiated mice. The IGF2BP3-overexpressing CD45.2 cells had a clear advantage over the MIG- or MIG-HOXA9-expressing cells in engraftment in the peripheral bleeds over time (Figure 5, D and E). Harvesting of the $\mathrm{BM}$ revealed that IGF2BP3 conferred a competitive advantage to cells in the BM (Figure 5F). This was also reflected in the thymus (Figure 5G). This corroborates our earlier data showing IGF2BP3 overexpression (Figure $5 \mathrm{H}$ ) leading to an increase in BM progenitor numbers as well as proliferation rate.

iCLIP identifies the IGF2BP3-RNA interactome in B-ALL cells. The molecular basis of the action of RBPs has recently been investigated using iCLIP and high throughput sequencing. To gain insight into the role of IGF2BP3 in cell growth and MLL-driven leukemogenesis, we performed an iCLIP assay with this protein. iCLIP exploits the photoreactivity of nucleic acid and protein residues and nuclease fragmentation of protein-bound transcripts to capture protein-RNA interactions occurring in situ. Antibodies against IGF2BP3 were used to immunoprecipitate protein-RNA complexes from control or UV-irradiated RS4;11 and REH cells (input shown in Supplemental Figure 5, A and C). As expected, the immunoprecipitated material was antibody dependent and UV dependent, and the electrophoretic mobility of the complex was nuclease sensitive, as predicted for a protein-RNA complex (Supplemental Figure 5, B and D). Coprecipitated RNA was converted to cDNA libraries (Supplemental Figure 5E) and subject to high throughput sequencing. After accounting for PCR duplications, we obtained about 1 million reads per replicate, of which $>70 \%$ mapped uniquely to the human genome (Supplemental Table 1). Replicate iCLIP sequences from both RS4;11 and REH cells were highly reproducible (Supplemental Figure 6, D and E). Compared with iCLIP cross link sites for heterogeneous ribonucleoprotein A1 (hnRNPA1; HEK cells) and with simulated data drawn randomly from the genome, IGF2BP3 cross-link sites, located at the 5 ' end of the iCLIP sequences, were enriched in exons (Figure 6A).

We identified peaks using a negative binomial model (see Methods) in each biological replicate from RS4;11 and REH cells. In total, 849 peaks in 669 genes and 1,937 peaks in 1,149 genes were identified in REH and RS4;11 iCLIP experiments, respectively. Of the peaks called within mRNA sequences, the majority were located within the $3^{\prime}$ UTRs (Figure 6B and Supplemental Figure $\left.6, \mathrm{~A}^{-} \mathrm{C}\right)$. A search for sequence specificity in cross-linked regions revealed an 8- to 16-fold enrichment of GCAC tetramercontaining motifs over background in both the REH and RS4;11 datasets (Figure 6C). Given the apparent bias of IGF2BP3 binding sites in 3'UTR of target transcripts, we investigated the positional bias of cross-linking sites at a single nucleotide resolution relative to mRNA stop codons. The cross-link density of IGF2BP3 differed from hnRNPA1 in the 3'UTR, reaching its apex just downstream of the stop codon. These data suggested that IGF2BP3 binding sites within the 3'UTR specifically target sequences close to the stop codon in both B-ALL cell lines (Figure 6D).

Based on the distribution of IGF2BP3 cross-linking sites within 3'UTRs (Figure 6D), we hypothesized that IGF2BP3 binding sites may overlap with cis-regulatory features associated with 3'UTRmediated gene regulation. To investigate this possibility, we examined the distribution of IGF2BP3 cross-linking sites relative to miR target sites in the 2 different cell lines. After correcting for the uniform background distribution of simulated cross-link sites, we found that IGF2BP3 cross-link density in both REH and RS4;11 cell lines is highly enriched within a 25-bp window centered on predicted miR target sequences (Figure 6E). By contrast, the density of hnRNPA1 cross-linking sites were uniformly distributed relative to miR target sites. Among the genes with a strong signal by iCLIP-sequencing (CLIP-Seq) were CDK6 and MYC. To confirm our findings demonstrating interaction of IGF2BP3 with these 2 targets by iCLIP (Figure 6, F and G), we utilized RNA immunoprecipitation (RIP; Figure $6 \mathrm{H}$ ), which showed enrichment of MYC and CDK6 (Figure 6I) in IGF2BP3 RIPs over mouse IgG control. These data demonstrate, for the first time to our knowledge, a comprehensive IGF2BP3 RNA interaction site atlas from human leukemia 
A

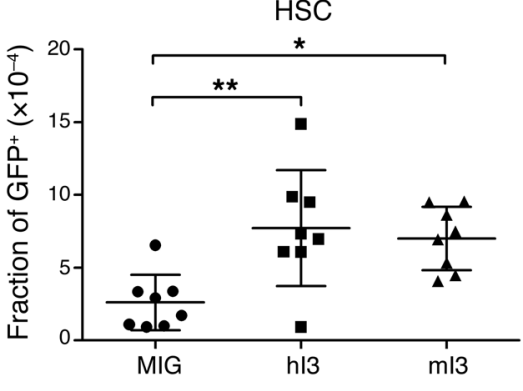

B

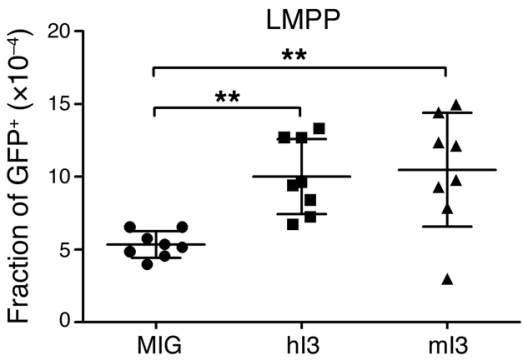

C

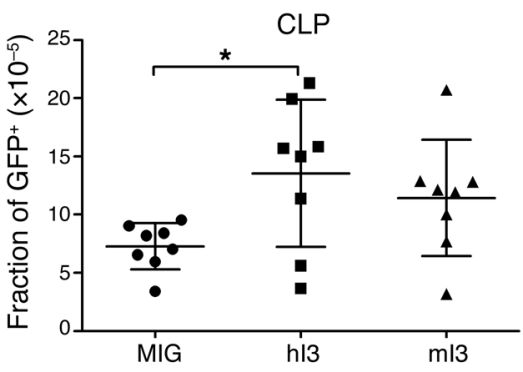

D

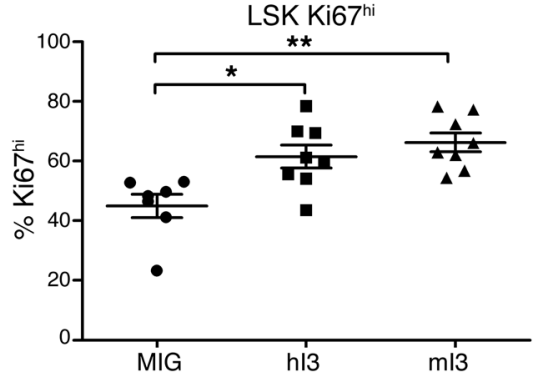

E

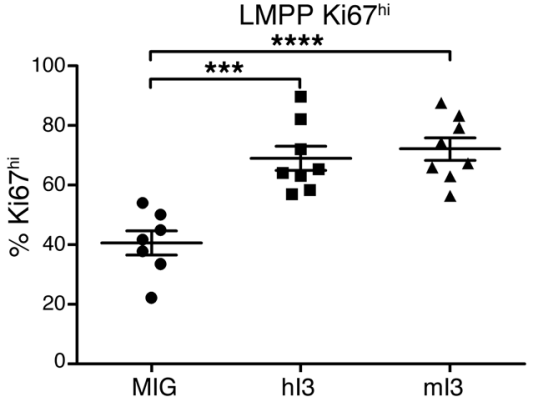

MIG
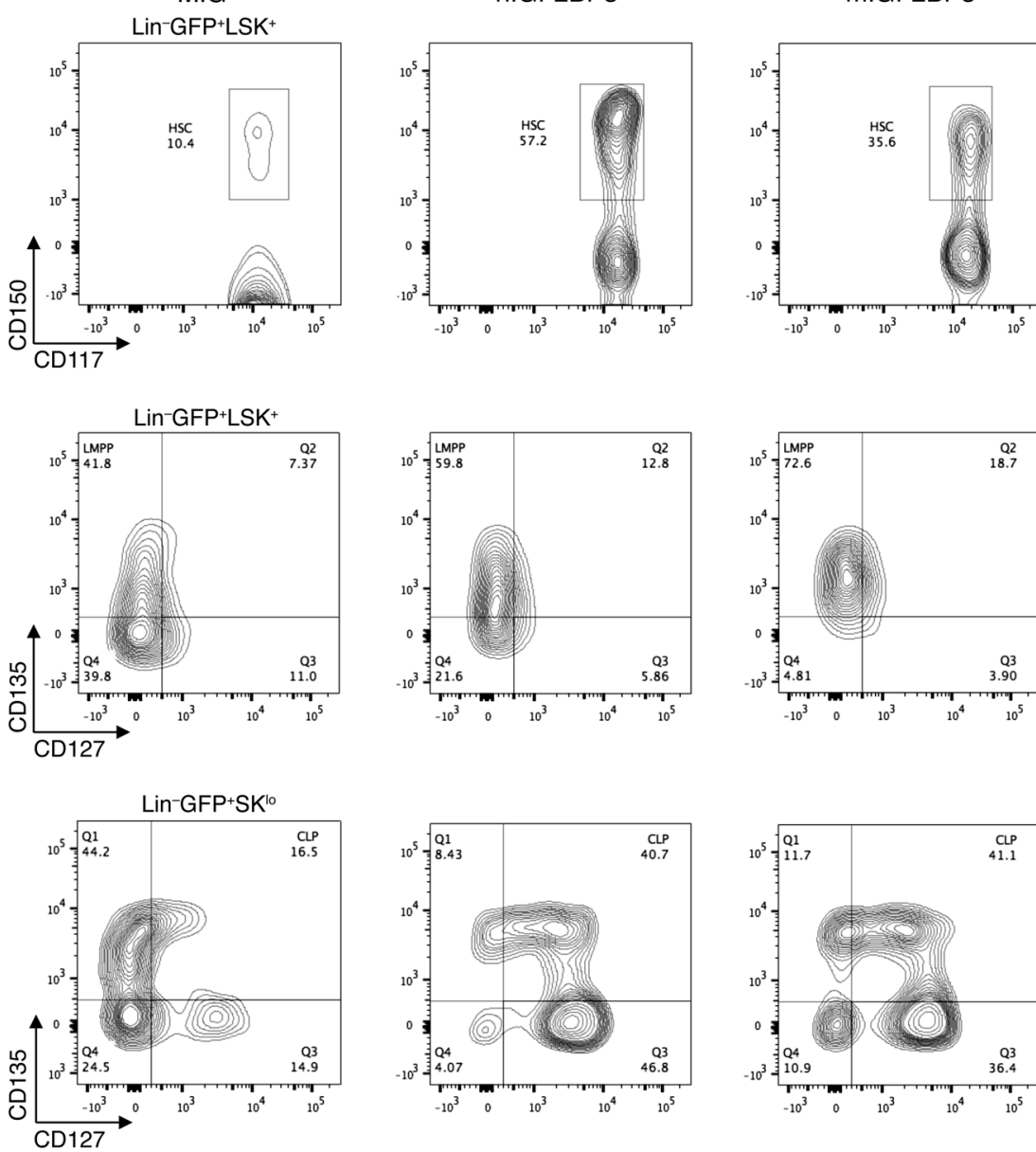

mIGF2BP3

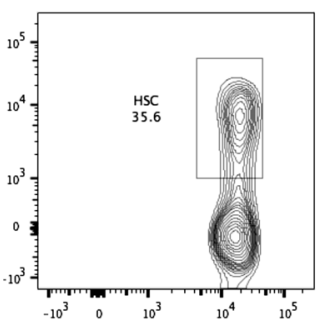

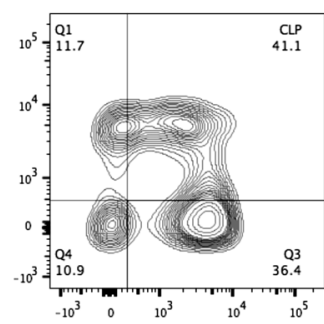
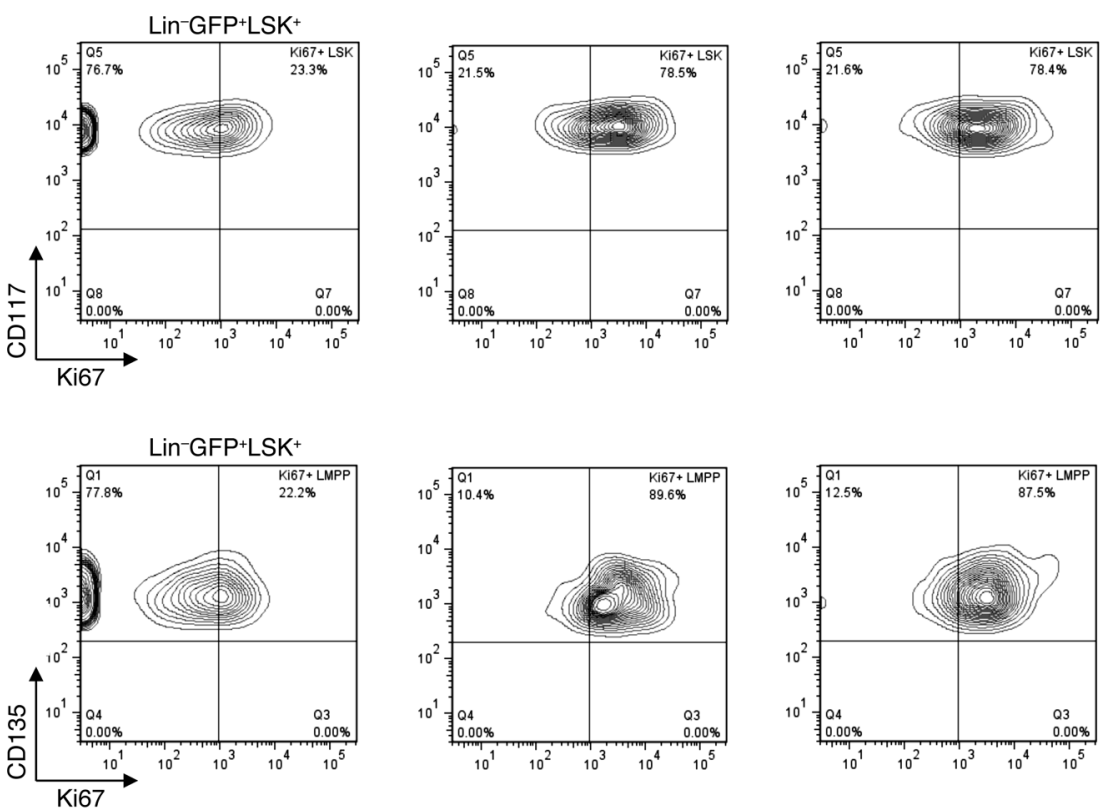

Figure 4. Analysis of BM progenitor populations from IGF2BP3-overexpressing mice. (A) Enumeration (left panel) and representative flow cytometry histograms to define HSCs from control vector- (second panel from left), human IGF2BP3- (second from right), and murine IGF2BP3-overexpressing mice (right panel). (B and C) Analysis for LMPPs and CLPs from mice noted as in A. Statistically significant differences were found in LMPPs and CLPs. (D) Intracellular Ki67 staining and FACS-based analyses, depicted in the same manner, with enumeration on the left hand side, within the LSK population enriched for HSCs. Significant differences in the high Ki67-expressing population were found. (E) Intracellular Ki67 staining and FACS analysis of proliferation in the LMPP population shows significant differences in the proliferative fraction. All comparisons used one-way ANOVA followed by Bonferroni's test. ${ }^{*} P<0.05$; ${ }^{* *} P<0.01 ;{ }^{* *} P<0.001 ;{ }^{* * *} P<0.0001$. LSK, Lin $-S c a 1{ }^{\mathrm{hi}} \mathrm{C}-\mathrm{Kit}^{\mathrm{hi}}$. Three separate BMT experiments were completed for validation. Data represent mean \pm SD. See also Supplemental Figure 3. hl3, human IGF2BP3; mI3, murine IGF2BP3. 
A
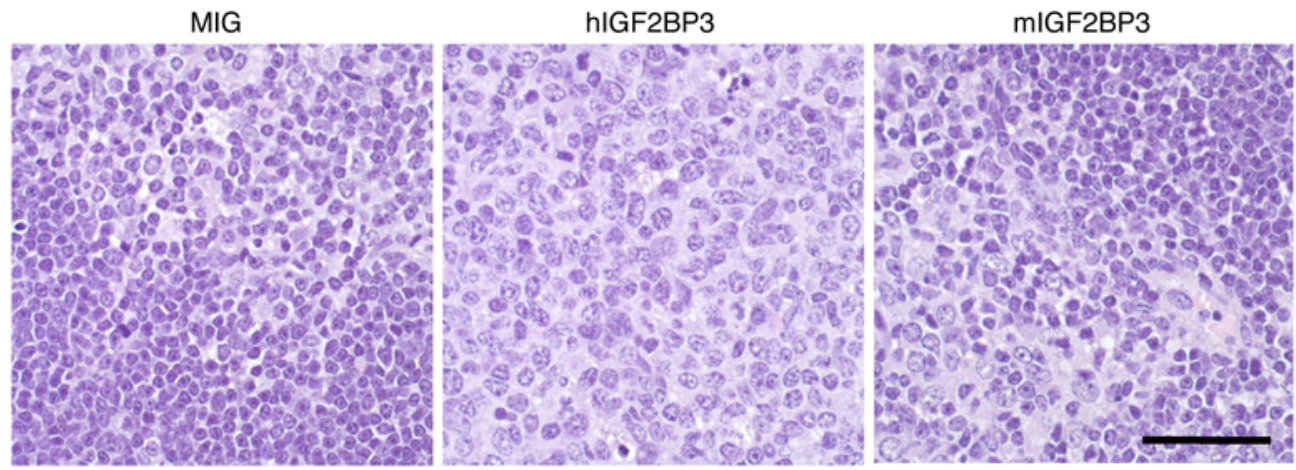

B
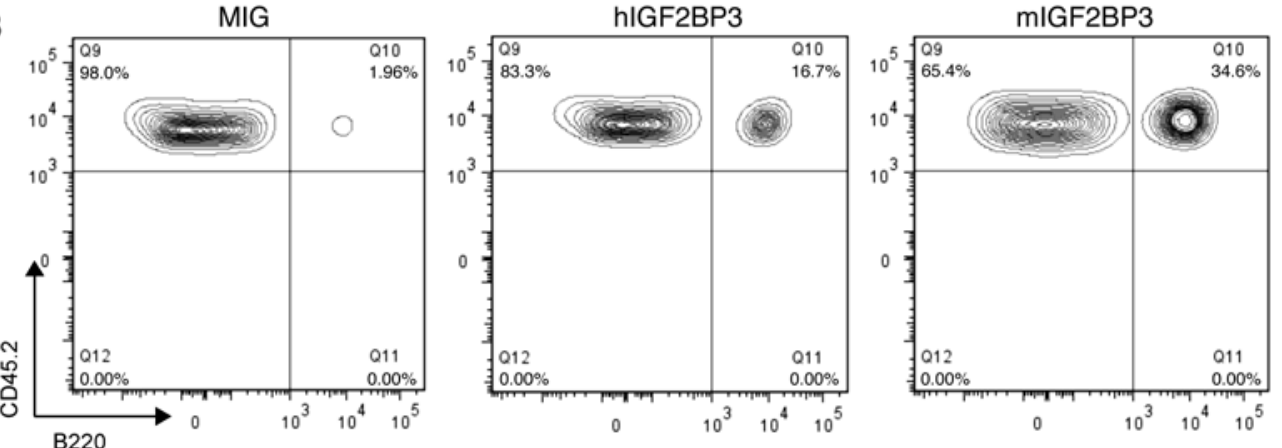

C

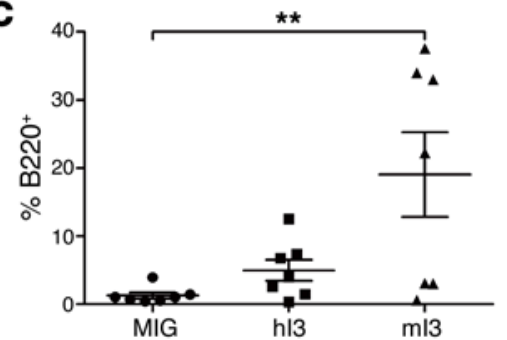

D

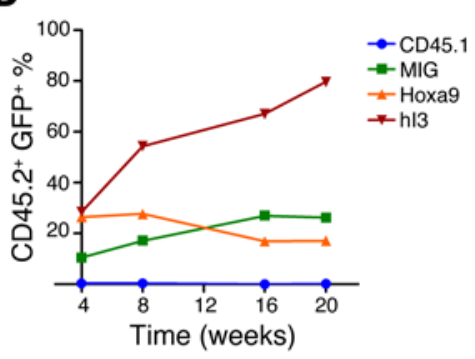

$\mathbf{F}$

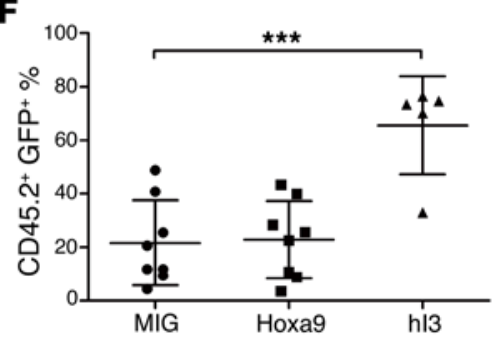

G

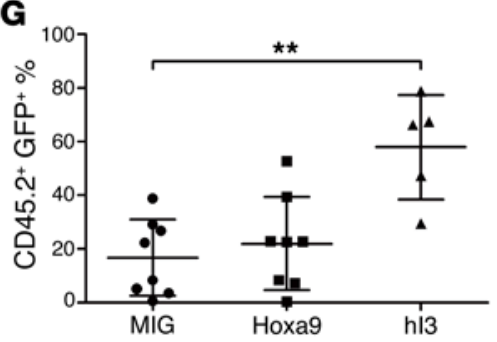

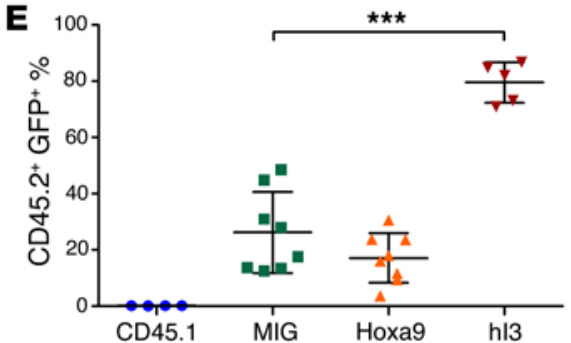

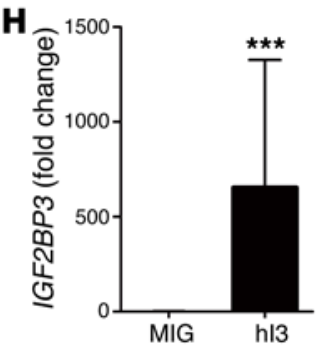

Figure 5. Analysis of thymic cellular composition and competitive repopulation advantage from IGF2BP3-overexpressing mice. (A) Histologic images of thymic sections from mice with enforced expression of ICF2BP3. H\&E staining. Scale bar: $40 \mu \mathrm{m}$. (B and C) Representative FACS plots and enumeration showing an increase in $B 220^{+}$cells in the thymus of mice with enforced expression (one-way ANOVA with Bonferroni's test; ${ }^{* *} P<0.01$ ). See also Supplemental Figure 4. $n=8$ for all 3 groups. Three separate BMT experiments were completed for validation. (D-H) Competitive repopulation study. (D) Quantitation of GFP expression in the PB between 4 and 20 weeks after transplant in competitive repopulation study of IGF2BP3. (E-G) FACS of PB (E), BM (F), and thymus (G) done at 20 weeks after BMT, showing CD45.2 and GFP positivity (one-way ANOVA followed by Bonferroni's test; ${ }^{* *} P<0.01$, $\left.{ }^{* * *} P<0.001\right)$. (H) qPCR confirmation of overexpression of IGF2BP3 in mouse BM ( $t$ test; $\left.{ }^{* * *} P=0.0006\right) . n=8$ (MIC), $n=8$ (Hoxa9), $n=5$ (hI3), and $n=4$ (100\% CD45.1). Competitive repopulation study was completed $3 \times$ for validation. Data represent mean \pm SD. hI3, human IGF2BP3; mI3, murine IGF2BP3; PB, peripheral blood.

cells. This extensive interaction map reveals a strong preference of IGF2BP3 binding nonuniformly to $3^{\prime} \mathrm{UTR}$ s with a preference for a GCAC-rich consensus motif near miR target sites.

IGF2BP3 modulates the expression of its targets. The ENRICHR tool (27) was used to functionally classify IGF2BP3 mRNA targets in REH and RS4;11 cells. In both cell lines, we found that target transcripts were enriched for KEGG pathways related to ribosome biogenesis and translation (Supplemental Table 2). By contrast, transcripts classified by ENRICHR as involved in pathogenic $E$. coli infection, and perhaps most importantly, chronic myeloid 

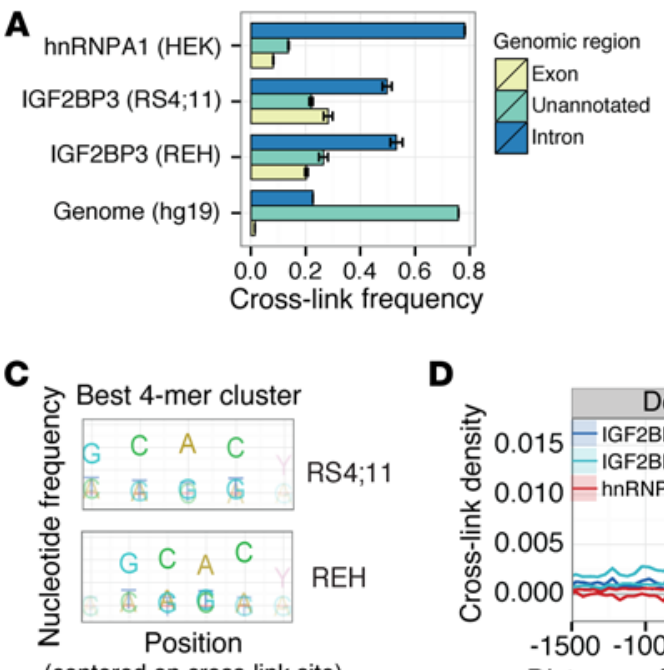

(centered on cross-link site)

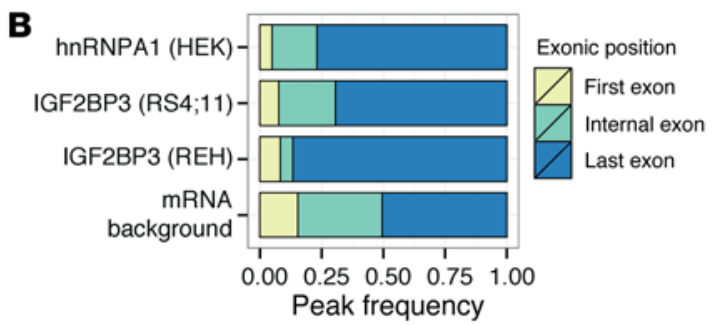

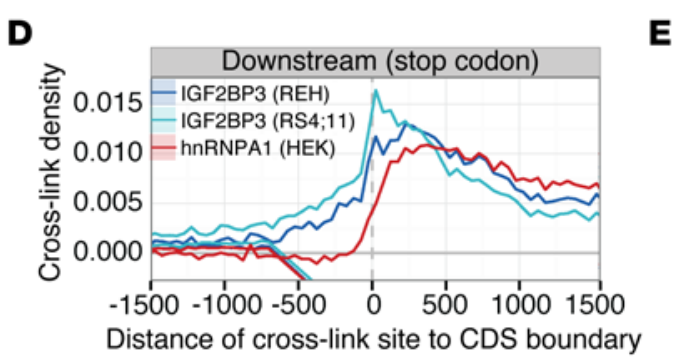
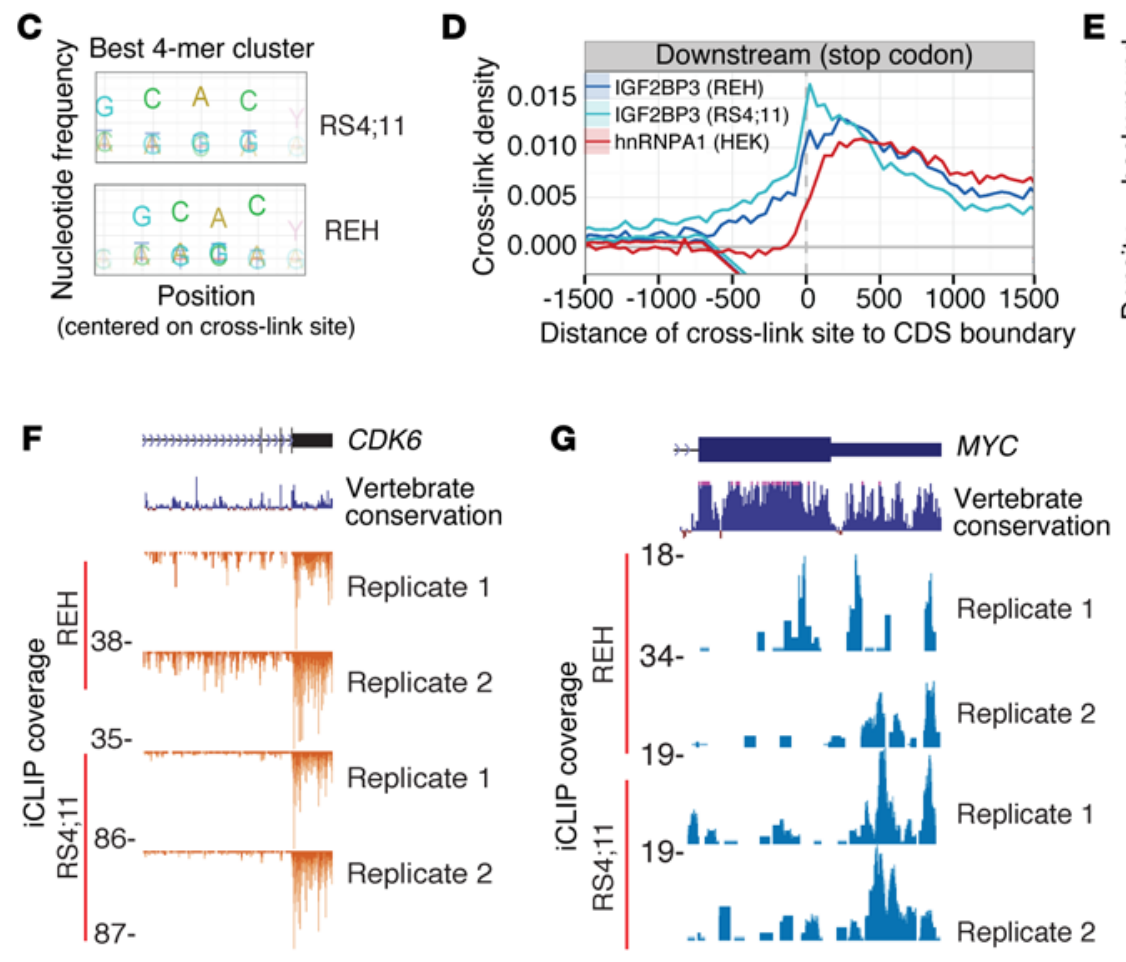
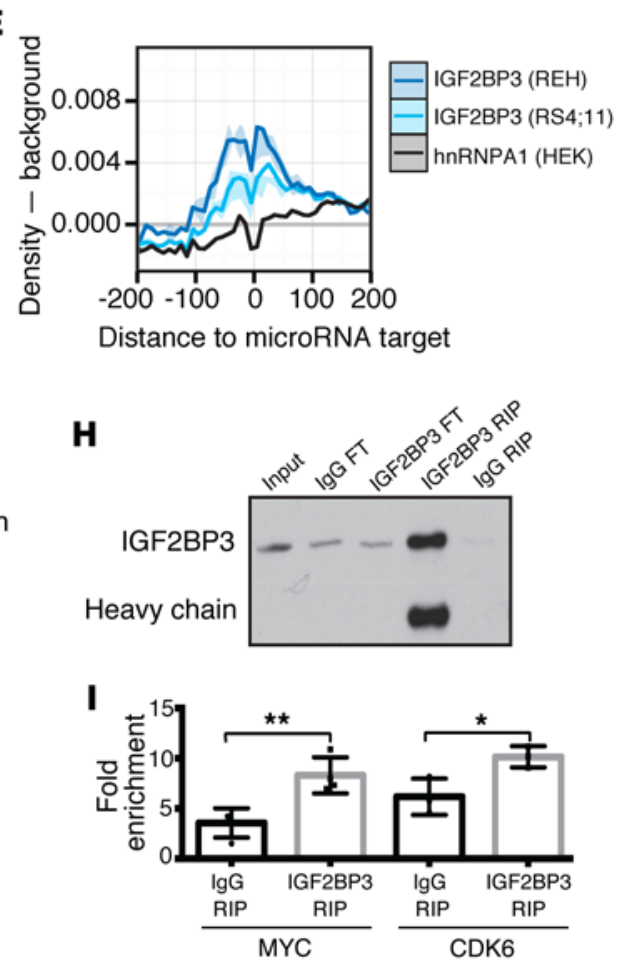

Figure 6. iCLIP analysis of IGF2BP3 in human leukemia cell lines. (A) Proportion of IGF2BP3 (REH and RS4;11 cells), hnRNPA1(HEK cells), and simulated (Genome) cross-linking sites observed in exons, introns, or unannotated regions of the human genome. (B) Proportion of ICF2BP3 (REH and RS4;11 cells), hnRNPA1 (HEK cells), and simulated (mRNA background) binding sites in coding and noncoding exons. (C) Tetramer sequence enrichment at IGF2BP3cross-linking sites in RS4;11 and REH cells (upper and lower panel, respectively). (D) IGF2BP3 (REH, RS4;11) and hnRNPA1 (HEK cells) cross-link site density relative to termination codons. (E) IGF2BP3 cross-linking density from REH (dark blue line) and RS4;11 (light blue line) cell lines mapped relative to annotated miR target sites. hnRNPA1 (black line) cross-linking sites from HEK293 cells are included as a control. (F and G) UCSC Genome Browser snapshot of the CDK6 and MYC 3'UTR loci, respectively. Each panel shows the exon-intron structure of the gene, sequence conservation across vertebrate species, and unique read coverage from 2 iCLIP replicates from each cell line. The maximum number of reads at each position is indicated to the left of each histogram. See also Supplemental Figures 5 and 6. (H) Western blot of protein samples from IGF2BP3 RIP. Input refers to RS4;11 cell lysate used for immunoprecipitation. FT is flowthrough of immunoprecipitation from either control (mouse IgG) or IGF2BP3 RIP. RIP is RNA immunoprecipitation from control (mouse IgG) or $\alpha$-IGF2BP3 antibody (D-7). (I) Scatter bar plots comparing the fold-enrichment for $M Y C\left(n=4, t\right.$ test; $\left.{ }^{* *} P<0.01\right)$ and CDK6 $(n=3$, $t$ test; ${ }^{*} P<0.05$ ) in control (mouse IgG) and $\alpha$-IGF2BP3 antibody RNA immunoprecipitations. Levels of MYC and CDK6 are normalized to input levels from total RNA with $18 \mathrm{~s}$ rRNA as reference.

leukemia (CML) were enriched in RS4;11 but not the REH dataset (Supplemental Table 2). Hence, we wanted to further explore the functional consequences of IGF2BP3 binding on gene expression at the global level. To determine if IGF2BP3 iCLIP targets (Supplemental Table 3) are regulated by IGF2BP3 expression levels, we performed RNA-Seq on control and IGF2BP3-depleted RS4;11 cells (Supplemental Table 4). Cross-validation of genes differentially expressed by at least 1.5 -fold with RS4;11-specific IGF2BP3 iCLIP targets found 216 common genes. Of these targets, the majority showed decreased expression of IGF2BP3 iCLIP targets with IGF2BP3 depletion (157 decreased vs. 59 increased; Figure 7A). Using the ENRICHR tool to classify the common genes cohort, OMIM disease gene ontology analysis (GO analysis) revealed genes associated with leukemogenesis (Figure 7A; black circles), including CDK6 and MYC. GO analysis for downregulated IGF2BP3 iCLIP targets revealed genes associated with posttranscriptional control, hematopoietic cell differentiation, and chromatin modification (Figure 7C). KEGG Pathway analysis also suggested these targets are involved in cell cycle and a variety of cancer pathways (Figure 7C). By contrast, the upregulated IGF2BP3 iCLIP targets revealed genes primarily associated with translation and protein localization (Figure 7B).

$C D K 6$ and MYC $m R N A$ s are targets of IGF2BP3 in vitro and in vivo. Among the many IGF2BP3 mRNA targets, CDK6 and MYC 
A Overlap of IGF2BP3 iCLIP targets and differentially expressed RNA transcripts in RS4;11 cells

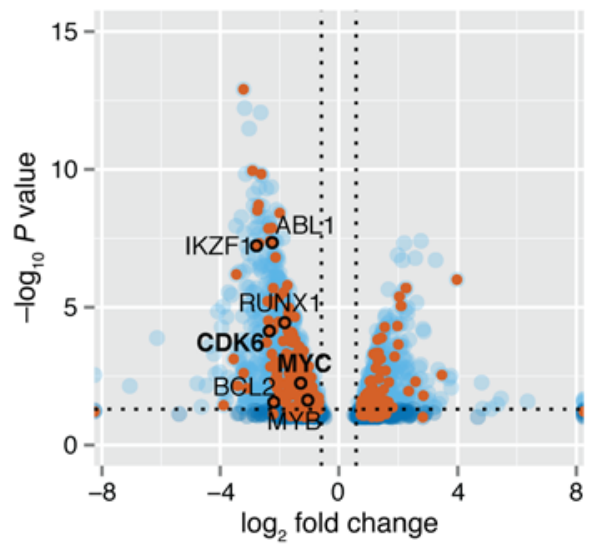

Transcript targets

Differentially-expressed transcripts

- Differentially-expressed + iCLIP target

B

RNA-seq/iCLIP overlap in genes showing increased expression with IGF2BP3 KD
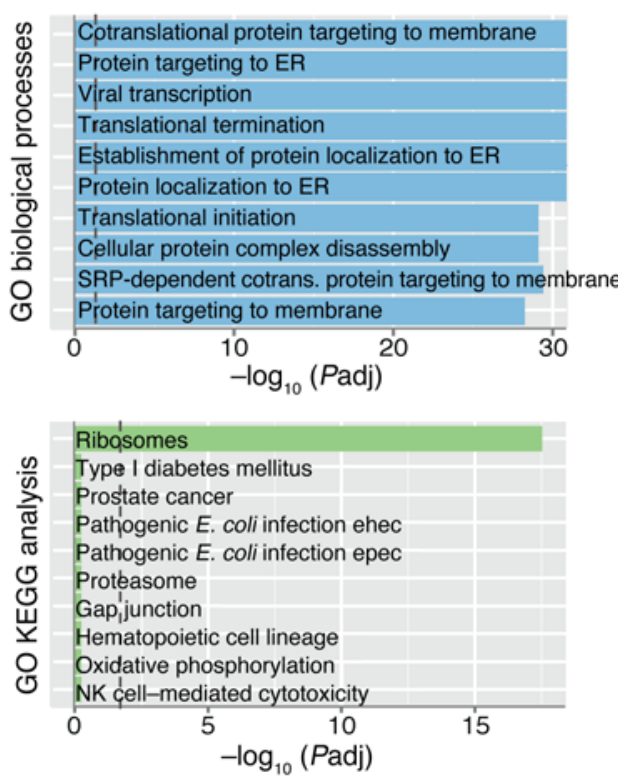

C showing decreased expression with IGF2BP3 KD
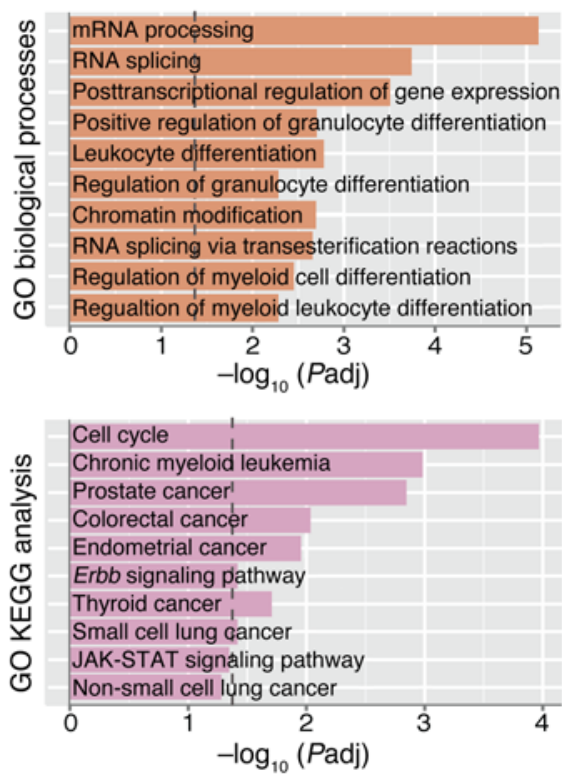

Figure 7. Cross-validation of IGF2BP3 iCLIP targets with IGF2BP3-sensitive differentially expressed genes. (A) Volcano plot of differentially expressed genes (blue dots) determined using DESeq analysis on RNA-Seq samples from control and IGF2BP3 knockdown RS4;11 cells (as described in Figure 2). Differentially expressed genes identified as IGF2BP3 targets by iCLIP are highlighted (orange dots). Dots demarcated by black outlines are leukemogenic genes by $\subset 0$ analysis of OMIM-associated disease pathways. Dotted lines represent 1.5-fold-change in expression (vertical lines) and $P<0.05$ cutoff (horizontal line). (B and C) $\mathrm{CO}$ analysis of gene subgroups showing increased expression (B) and decreased expression (C) with IGF2BP3 knockdown using ENRICHR gene list enrichment analysis webtool. Term lists used in this analysis were $\mathrm{GO}$ Biological_Processes and KEGG to determine enriched processes and pathways from our cross-validated list of 269 IGF2BP3-targeted and -sensitive genes. Vertical dotted lines represent $P$ value cutoff $(P<0.05)$. KD, knockdown. are very important in the pathogenesis of MLL-translocated B-ALL $(6,7)$. In our patient data set, MLL-translocated cases of B-ALL demonstrated high levels of these genes, in line with their proposed role as targets bound by and regulated by IGF2BP3 (Supplemental Figure 1, A and B). To test the hypothesis that IGF2BP3 posttranscriptionally regulates the expression of CDK6 and MYC via binding sites in their 3'UTR, we generated a series of vectors using a dual-luciferase reporter system containing the respective UTRs (Figure 8A). The CDK6 3'UTR (10 kb) was cloned in 5 separate pieces (CDK6-1 to CDK6-5). Cotransfection of the luciferase vectors with IGF2BP3 resulted in increased luciferase activity in CDK6-3 to CDK6-5, as well as the MYC 3'UTR reporters (Figure $8 \mathrm{~B})$. This, along with the iCLIP and RNA-Seq data, confirms IGF2BP3 binding to the 3'UTRs of these genes may stabilize the mRNA and/or enhance translation. Mutation of one of the binding sites within the MYC $3^{\prime} \mathrm{UTR}$, designated MYC $\Delta 3$, resulted in a small but reproducible attenuation of IGF2BP3-dependent enhancement of the MYC 3'UTR reporter (Figure 8C). However, mutation of individual IGF2BP3 binding sites in the CDK6 $3^{\prime} \mathrm{UTR}$, followed by IGF2BP3 overexpression and a luciferase assay, did not show any significant difference (data not shown). These findings may result from one of the following possibilities: (i) binding may not be sufficient for alterations of gene expression, or (ii) cooperative binding at multiple sites may be required for an effect on gene expression. Indeed, some parallels have been seen in the case of miRs, where increased repression is seen in targets with multiple binding sites (28). Nonetheless, IGF2BP3 binding would be predicted to lead to increased levels of CDK6 and MYC in cells overexpressing IGF2BP3 and to decreased levels in knockdown cells.

To elucidate whether IGF2BP3 targets CDK6 and MYC in vivo, we performed intracellular staining in BM cells from mice with enforced IGF2BP3 expression and measured mean fluorescence intensity (MFI) by flow cytometry. $\mathrm{BM} \mathrm{GFP}^{+}$cells derived from human IGF2BP3-expressing mice had increased MFI for CDK6 and MYC (Figure 8, D and F). As a control, GFP cells of both groups did not show any difference (Figure 8, E and G). To complement these in vivo data, we analyzed CDK6 and MYC protein levels in cell lines where IGF2BP3 was knocked down using 
A

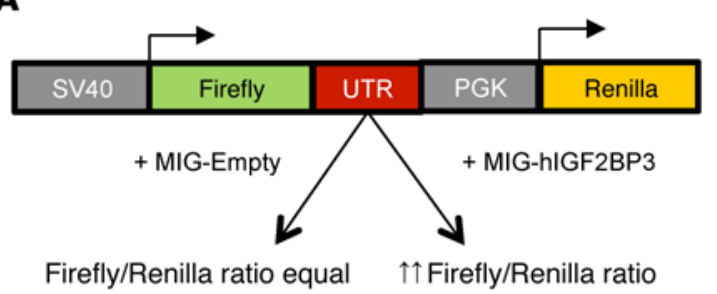

B

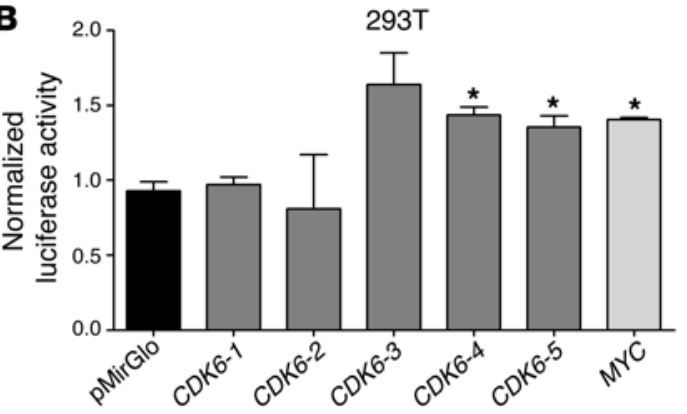

C

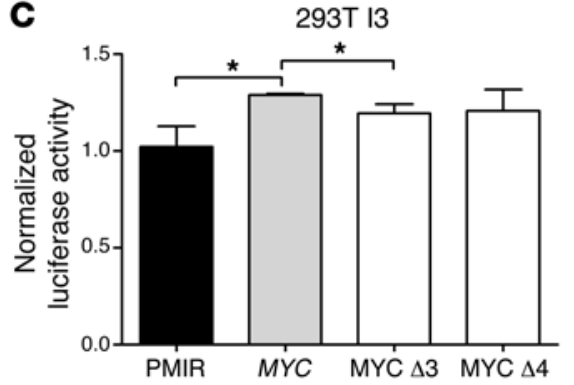

$\mathbf{F}$

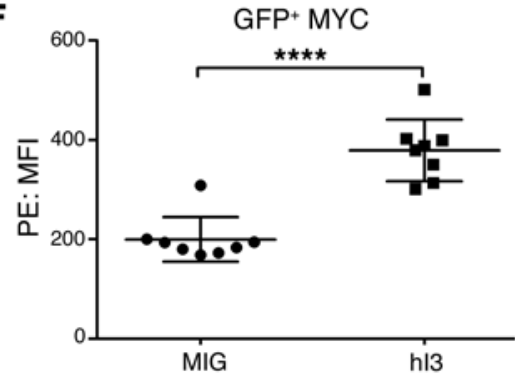

D

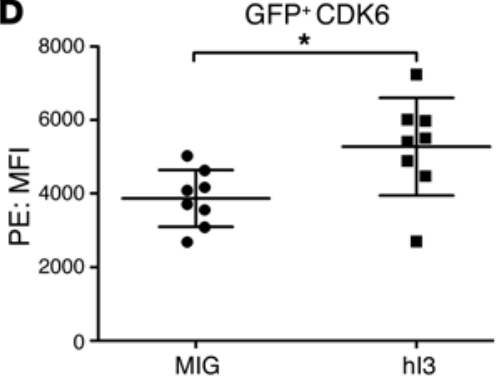

G

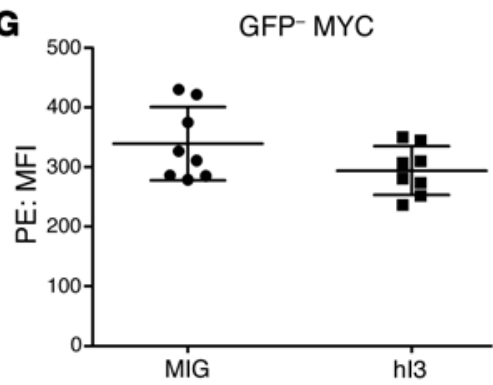

E

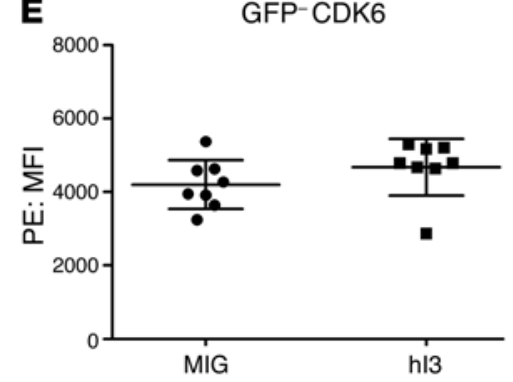

H

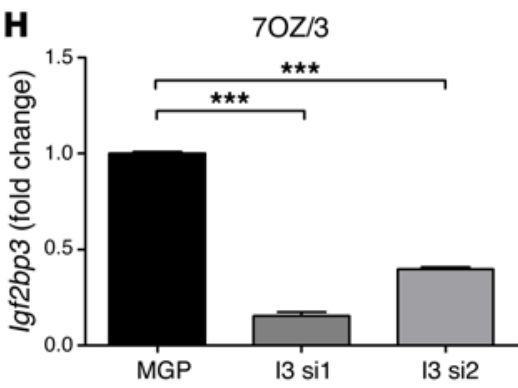

I

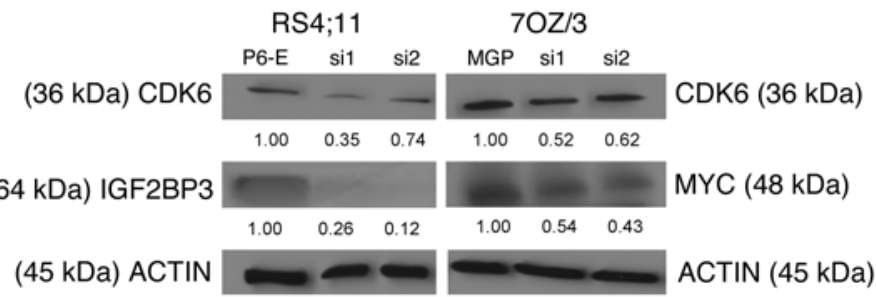

Figure 8. CDK6 and MYC are targeted by IGF2BP3. (A) Schematic of the luciferase assay used. (B) Luciferase assay showing targeting of the CDK6 and MYC 3'UTRs by IGF2BP3 ( $t$ test ${ }^{*} P=0.05 ; P=0.0250$ CDK6-4, $P=0.0475$ CDK6-5, $P=0.0165$ MYC). (C) Deletion of the MYC $3^{\prime} U$ TR binding sites of ICF2BP3 led to modest but significantly decreased luciferase activity ( $t$ test ${ }^{*} P=0.05 ; P=0.0120 \mathrm{MYC}, P=0.0294$ MYC $\triangle 3$ ). (D and E) CDK6 analysis of BM progenitors shows a significantly increased amount of CDK6 protein in the GFP+ BM cells ( $t$ test; $\left.{ }^{*} P=0.0213\right)$ (D) but not in the GFP- $(\mathbf{E})$ cells. $(\mathbf{F}$ and $\mathbf{C})$ Intracellular staining for MYC reveals significantly increased levels in the GFP+ BM cells after IGF2BP3-enforced expression ( $t$ test; $\left.{ }^{* * * *} P<0.0001\right)(\mathbf{F})$ but not in the GFP- $(\mathbf{G})$ cells. $n=8$ for all 3 groups. ( $\mathbf{H}$ and $\mathbf{I})$ After Igf2bp3 knockdown in 70Z/3 cells ( $t$ test IGF2BP3 si1 and si2, respectively; ${ }^{* * *} P=0.0007$, $\left.{ }^{* * *} P=0.0005\right)$, there is reduced expression of CDK6 and MYC protein $(\mathbf{H})$. In the RS4;11 cell line, Western blot confirmed knockdown of IGF2BP3 protein and reduced expression of CDK6 protein (I). Experiments were conducted $3 \times$ for validation. Data represent mean \pm SD. See also Supplemental Figure 7. hl3, human ICF2BP3; PE, phycoerythrin.

siRNAs previously. There was a substantial decrease in CDK6 protein levels in RS4;11 cells after IGF2BP3 knockdown (Figure 8I). Similarly, Igf $2 b p 3$ knockdown in the murine pre-B 7OZ/3 cell line led to a reduction in CDK6 and MYC protein (Figure 8, $\mathrm{H}$ and I). These findings are in agreement with the RNA-Seq data and demonstrate a conserved function for IGF2BP3 (Figure 7A).

At the mRNA level, IGF2BP3 overexpression led to a slight but significant increase in $M y c$ mRNA levels, but not in $C d k 6$, in bulk
BM (Supplemental Figure 7, A and B). It is possible that an increase in $C d k 6$ mRNA may not be detected due to the heterogeneity of the BM cells or because effects on mRNA stability by IGF2BP3 may be mRNA specific. Similarly, murine 7OZ/3 cells showed a modest increase in the $C d k 6$ mRNA levels when murine IGF2BP3 was overexpressed (Supplemental Figure 7, C-E). These findings corroborate IGF2BP3 binding and subsequent translational augmentation of these target genes. Overall, these experiments 
A
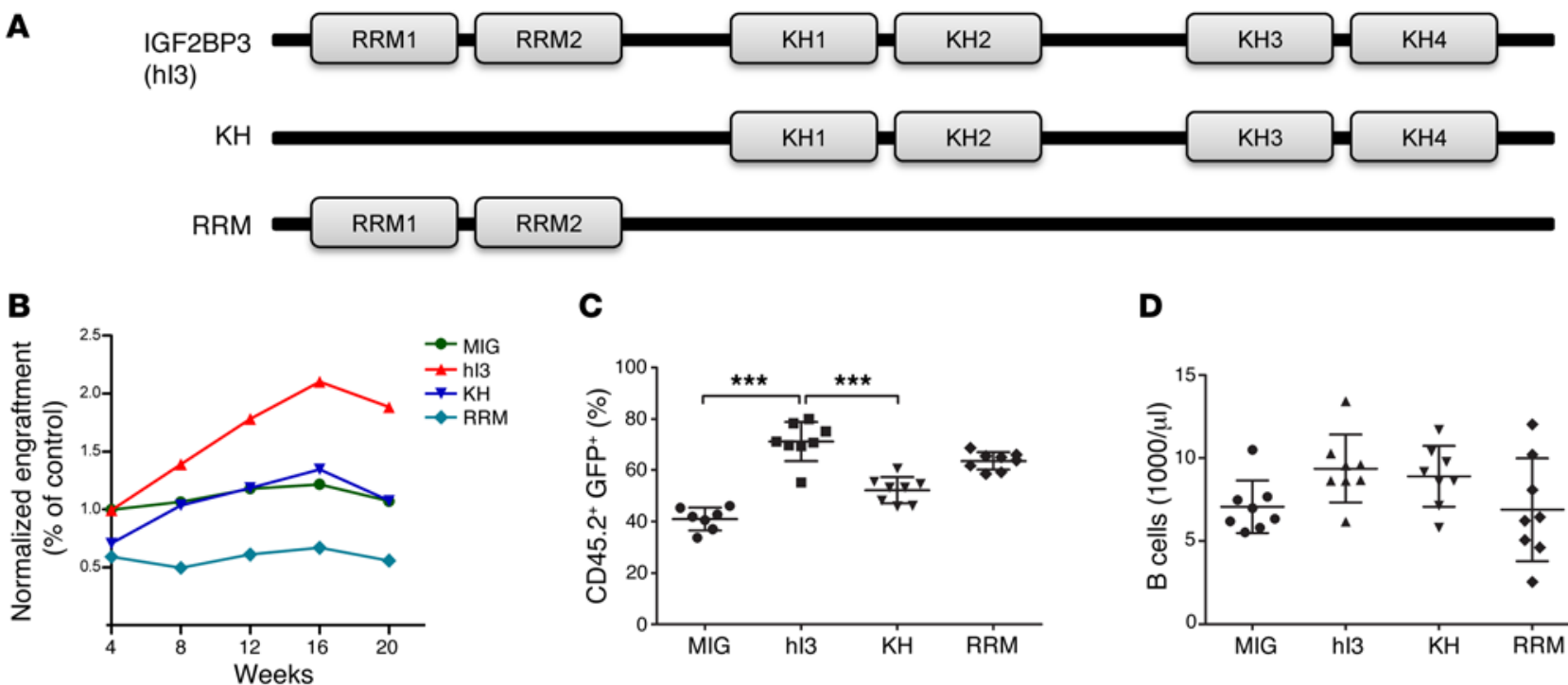

C

D

E

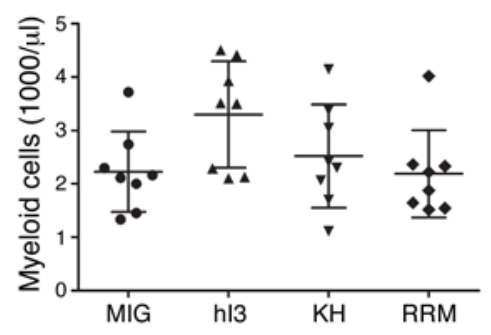

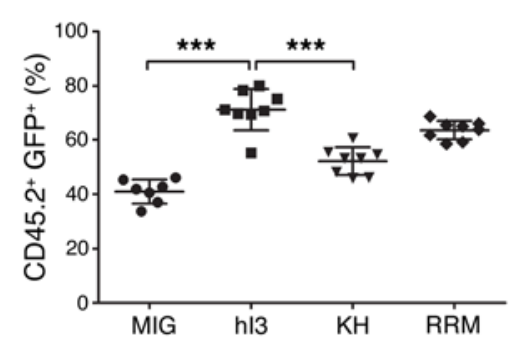

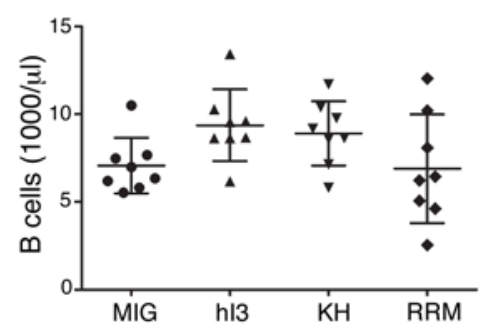

$\mathbf{F}$

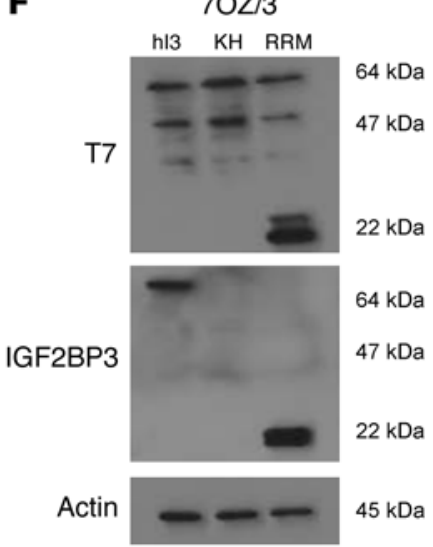

G

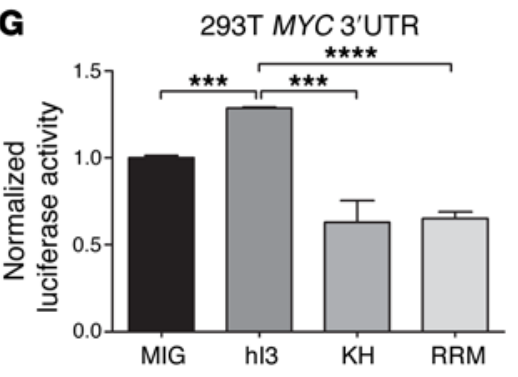

Figure 9. Expression of IGF2BP3 RNA-binding domain mutants in vivo and in vitro. (A) Schematic of IGF2BP3 with its binding domains and the respective mutants (KH and RRM). (B) Time course of normalized engraftment to MIG in PB between 4 and 20 weeks after transplant. (C) FACS of PB done at 4 weeks after BMT, showing CD45.2 and GFP positivity (one-way ANOVA followed by Bonferroni's test; ${ }^{* * *} P<0.001$ ). (D) B cells in PB 16 weeks after transplant. (E) Myeloid cells in PB 12 weeks after transplant. $n=8$ for all groups. Mutant BMT experiment was completed twice for validation. (F) Western blot confirmed expression of IGF2BP3 (64 kDa), KH (47 kDa), and RRM (22 kDa) proteins in 70Z/3 using anti-T7 (top panel) and anti-IGF2BP3 (bottom) antibodies. Actin used as a loading control. (G) Luciferase assay shows increased luciferase activity for the MYC $3^{\prime} U T R$ when cotransfected with hl3 and decreased luciferase activity for the MYC $3^{\prime} \mathrm{UTR}$ when cotransfected with $\mathrm{KH}$ and RRM mutants ( $t$ test hl3, K,H and RRM; ${ }^{* * *} P<0.001 ;{ }^{* * *} P<0.0001$ ). Experiment was completed $3 \times$. Data represent mean \pm SD. hl3, human IGF2BP3; PB, peripheral blood.

demonstrate targeting of CDK6 and MYC by IGF2BP3 in a variety of systems, and that may underlie the observed phenotypic effects of enforced expression.

Mutated IGF2BP3 does not increase hematopoietic progenitor numbers in vivo. IGF2BP3 has 6 RNA-binding domains: 2 RNA recognition motif domain (RRM) and $4 \mathrm{~K}$ homology domain $(\mathrm{KH})$ domains that are predicted to mediate the RNA-binding function of IGF2BP3. We created 2 deletion mutants: the $\mathrm{KH}$ mutant, containing only the $4 \mathrm{KH}$ domains and devoid of both the RRM domains, and the RRM mutant, containing the 2 RRM domains and lacking the $4 \mathrm{KH}$ domains (Figure 9, A and F). A murine BM transplant was performed with MIG, WT IGF2BP3, $\mathrm{KH}$, and RRM mutants into lethally irradiated recipients. Unlike the WT protein, enforced expression of these mutant proteins failed to cause enhanced hematopoiesis or the skewing toward the B cell and myeloid lineages that we observed previously.
The mutant recipient mice showed reduced engraftment (Figure 9, B and C). B cell and myeloid cell counts in the periphery showed a trend toward normalization in IGF2BP3 mutant mice (Figure 9, D and E). A luciferase assay using the MYC $3^{\prime} \mathrm{UTR}$ and comparing WT and mutant IGF2BP3 demonstrated decreased luciferase activity, confirming the idea that these mutants no longer bind to and/or stabilize target mRNAs (Figure 9G). Interestingly, one study reported that the role of $\mathrm{KH}$ domains is not well understood in IGF2BP3, among the various IGF2BP family members (29). Although the precise determinants of RNA binding are not known, our findings provide an impetus to perform detailed structure-function analyses to elucidate domains and residues important for IGF2BP3 function. Together with our high throughput data, these experiments provide some of the first comprehensive studies that link the RNA binding function of this protein to organism-level phenotypes. 


\section{Discussion}

The molecular mechanism of leukemogenesis mediated by MLLfusion proteins is not completely understood, despite knowledge of the translocation and the resulting fusion proteins for 2 decades. Increasingly, it is recognized that secondary, nongenetic changes are necessary for elaboration of leukemia. For example, deregulation of epigenetic marks by DOT1L, which is recruited by the MLL-AF4 fusion protein, is a key oncogenic mechanism (30). In this study, we found that an RBP, IGF2BP3, is overexpressed in cases of B-ALL that carry a translocation of the MLL gene. We propose that posttranscriptional gene expression dysregulation may also play an important role in leukemogenesis. This is borne out by prior studies of another RBP, musashi-2, which demonstrated that it was highly expressed in acute myeloid leukemia and that its overexpression could collaborate with BCR-ABL to promote myeloid leukemogenesis (31). Another recent example is the HuR protein, which is upregulated in multiple epithelial malignancies and is known to stabilize its targets by binding to AU-rich elements within the 3'UTR of target mRNAs (32). Our findings here extend the repertoire of dysregulated $\mathrm{RBP}$ expression to B-ALL, providing new insights into this disease.

The mechanism of IGF2BP3 upregulation in MLL-AF4expressing leukemia is an important question. Our studies showed that a BET domain inhibitor could downregulate IGF2BP3 in RS4;11, and this is thought to specifically target MLL-mediated transcription at low doses (23). Previous reports have also shown IGF2BP3 to be upregulated after MLL-AF4 overexpression in murine BM cells (4). Interestingly, an indirect ChIP-Seq assay done in SEM cells (which carry the MLL-AF4 translocation) showed binding of the fusion protein to the genomic locus containing IGF2BP3 (33). Therefore, it is tempting to speculate that IGF2BP3 is a direct transcriptional target of MLL fusion proteins. However, IGF2BP3 is also overexpressed in most of the B-ALL cell lines tested, various mature B cell neoplasms (34-37), and a number of epithelial malignancies. Furthermore, differential regulation of this protein has been observed in B-ALL (38). Hence, the mechanism of its upregulation may include other oncogenic pathways.

Previously, knockdown of IGF2BP3 in epithelial cell lines has been shown to reduce cell proliferation and cause apoptosis $(18,37$, 39). We found that knockdown or deletion of IGF2BP3 by siRNA or by the CRISPR-Cas9 system led to reduced cell proliferation and increased apoptosis in RS4;11, an MLL-AF4-expressing cell line, and NALM6, another B-ALL cell line that shows high levels of IGF2BP3. These findings highlight the important role that posttranscriptional gene regulation can play in maintaining the malignant behavior of B-ALL cells. It will be of great interest to study whether B-ALL with low expression levels of IGF2BP3 demonstrate an altered RNA-binding repertoire, with an aim toward illuminating key mRNAs that mediate downstream effects of IGF2BP3.

To study the pathogenetic function of this protein, we also created the first in vivo model of IGF2BP3-enforced expression in the murine hematopoietic system. We found that IGF2BP3 increases the number of HSCs, LMPPs, and CLPs in the BM with a concomitant increase in the proliferation rate of HSCs and LMPPs. IGF2BP3 conferred a competitive reconstitution advantage and skewed mouse hematopoiesis toward the B cell and myeloid lineage in the periphery, with leukocytosis in the peripheral blood, atypical B cell infiltration into the thymic medulla, and increased myeloid cells in the spleen. Although the mice did not develop overt leukemia by 6 months after transplantation, these abnormal developmental features are similar to those seen early in MLLdriven leukemogenesis, which include the expansion of the B cell and myeloid lineage. Such IGF2BP3-driven effects on stem and progenitor cells and differentiation fates may extend beyond the hematopoietic system. IGF2BP3 can cause remodeling of the exocrine pancreas when specifically overexpressed in that tissue and is highly expressed in cancer stem cells/tumor-initiating cells in hepatocellular carcinoma $(40,41)$.

It has been known that IGF2BP3 targets mRNAs for IGF2, $C D 44$, and the transcription factor $\operatorname{HMGA2}(16,42)$. Further reports on HMGA2 and IGF2BPs have suggested that they may play a role in the self-renewal potential of fetal HSCs $(43,44)$. Let-7 miR has been known to inhibit tumor cell migration and invasion by targeting IGF2BP3 (45). Thus, IGF2BP3 may function in regulating both developmental and oncogenic processes. To examine the molecular mechanism behind the observed disruptions in cellular and hematopoietic homeostasis, we performed iCLIPSeq analyses. Remarkably, we identified numerous RNA targets of IGF2BP3 in B-ALL cell lines that were known targets of MLL, including CDK6 and MYC. CDK6 has recently been implicated as a highly important target in B-ALL, and inhibition of CDK6 may form the basis of a new therapeutic intervention in B-ALL (7). MYC is a quintessential oncogene, and its overexpression plays a direct, causative role in many B cell leukemias and in lymphoma. Reporter assays confirmed that the interaction of IGF2BP3 with the 3'UTRs of MYC and CDK6 are functionally important. IGF2BP3 with deletions of RNA-binding domains failed to bind and stabilize MYC mRNA in the luciferase reporter assay. However, when single IGF2BP3 binding sites were deleted in the MYC and CDK6 3'UTR, the majority of these deletions failed to reverse the phenotype in the reporter assay. The complexity of 3'UTR targeting is illustrated by the variety of RBPs and noncoding RNAs that have been reported to bind to the MYC and CDK6 3'UTRs (46-49). Hence, RBP action on its cognate targets is likely dose dependent and cooperative; multiple RBPs have to bind to the same 3'UTR at different locations to exert an effect. In vivo and in vitro targeting of CDK6 and MYC protein was confirmed in the mouse BM and leukemia cell lines, in both the loss- and gain-offunction settings. Hence, we have validated iCLIP as a powerful technique for uncovering disease-relevant targets. It is important to point out that there are likely mRNAs other than MYC and CDK6 that interact with IGF2BP3, including those we identify here, that play important roles in cellular proliferation and/or differentiation. This is illustrated by our attempt to rescue IGF2BP3 knockdown-mediated cell death by cotransducing RS4;11 cells with MYC constructs containing or lacking the $3^{\prime} \mathrm{UTR}$. MYC alone was not sufficient to rescue changes in the cell cycle engendered by the loss-of-function of IGF2BP3 (data not shown).

The iCLIP-Seq analyses provide a global view of the transcriptome regulated by IGF2BP3. Prior studies have demonstrated single or a few targets for this protein, but our work here shows several hundred mRNAs bound by this protein. To refine our list of possible transcripts, we combined this biochemical target identification with gene expression studies in cells with IGF2BP3 loss 
of function. Distinct sets of genes that were bound by IGF2BP3 showed upregulation or downregulation, with downregulated genes demonstrating enrichment for pathways related to cell cycle and leukemia. These findings suggest that IGF2BP3 upregulation in B-ALL serves to stabilize leukemogenic gene expression. Like other modes of posttranscriptional gene expression regulation, the actions of IGF2BP3 are dependent on the cellular transcription program. IGF2BP3 is itself induced by MLL-AF4, and it binds to and upregulates several mRNAs (e.g., CDK6 and MYC) that are also induced by MLL-AF4. In this way, IGF2BP3 may reinforce certain aspects of the gene expression program, thereby sustaining oncogenesis. Interestingly, however, enforced expression of this protein alone also led to proliferation of hematopoietic progenitor cells, suggesting that specificity of RNA stabilization and/ or translational enhancement can direct development and influence lineage choice and proliferation.

As oncogenic mutations and translocations are being catalogued via various high throughput sequencing approaches, it is also becoming apparent that single genetic abnormalities are insufficient to cause oncogenesis. Prior work has implicated nongenetic mechanisms, including epigenetic regulation, as key factors in the pathway to full-blown oncogenesis. Here, our studies have uncovered a posttranscriptional mechanism of stabilizing oncogenic gene products such as CDK6 and MYC. This mechanism requires further study and clarification and is likely to yield important insights into the nature of gene expression regulation in leukemogenesis. With targeted therapies emerging against CDK6 and MYC, it will become critical to consider the role of posttranscriptional mechanisms in regulating oncogene-mediated gene expression programs. Moreover, therapeutic avenues are also suggested by the current study, including the generation of sink RNAs to block the binding of IGF2BP3 to its targets or small molecule inhibitors of this protein designed to block its RNA-binding function. Given the expression of IGF2BP3 in many different types of cancer, it will be of great interest to define whether the repertoire of bound mRNAs is similar in tumors of distinct histogenesis and whether conserved oncogenic pathways can be targeted in hematologic and nonhematologic malignancies.

\section{Methods}

Patient samples, $\mathrm{CD} 19^{+}$cell isolation, and microarray data analysis. All procedures and protocols related to these have been previously described, and the microarray data set has been made publically available (NCBI's Gene Expression Omnibus [GEO GSE65647]) (21).

Apoptosis, proliferation, and cell cycle analysis. To measure cell proliferation, 2,000-4,000 cells per well were cultured in 96-well plates. MTS reagents were added according to the manufacturer's instructions (Promega CellTiter $96 \mathrm{AQ}_{\text {ueous }}$ Non-Radioactive Cell Proliferation Assay kit) and cells were incubated at $37^{\circ} \mathrm{C}, 5 \% \mathrm{CO}_{2}$ for 4 hours before absorbance was measured at $490 \mathrm{~nm}$. To measure apoptosis, cells were stained with APC-tagged annexin V and analyzed by flow cytometry. For cell cycle analysis, cells were fixed with $70 \%$ ethanol and stained with $1 \times$ propidium iodide solution in PBS and analyzed using flow cytometry.

qPCR. RNA collected from human samples was reverse transcribed using iScript reagent (Quanta BioSciences). RNA from cell lines was reverse transcribed using qScript (Quanta BioSciences).
qPCR was performed with the StepOne Plus Real-Time PCR System (Applied Biosystems) using PerfeCTa SYBR Green FastMix reagent (Quanta BioSciences). The qPCR primer sequences used are listed in Supplemental Table 5 .

Western blot. Cells were lysed in RIPA buffer (Boston BioProducts) supplemented with Halt Protease and Phosphatase Inhibitor Cocktail (Thermo Scientific). Equal amounts of protein lysate (as quantified by using bicinchoninic acid protein assay, BCA [Thermo Scientific]) were electrophoresed on a 5\%-12\% SDS-PAGE and electroblotted onto a nitrocellulose membrane. Antibodies used were c-MYC rabbit polyclonal (catalog 9402, Cell Signaling Technology), CDK6 rabbit monoclonal (catalog DCS83, Cell Signaling Technology), IGF2BP3 goat polyclonal (catalog sc-47893, Santa Cruz Biotechnology Inc.), T7 rabbit polyclonal (catalog AB3790, Millipore), and $\beta$-actin (cata$\log$ AC15, Sigma-Aldrich) mouse monoclonal. Secondary HRP-conjugated antibodies (Santa Cruz Biotechnology Inc.) and SuperSignal West Pico kit (Pierce Biotechnology) were used for enhanced chemiluminescence-based detection.

Cell culture, plasmids, and spin infection. mmu-miR-155 or hsamiR-21 formatted siRNAs were cloned between NotI and BamHI sites of a pHAGE6 lentiviral vector (pHAGE6-CMV-siRNA-UBC-ZsGreen) or between NotI and BamHI sites of a modified pHAGE6 vector downstream of GFP (CMV-GFP-siRNA-UBC-Puromycin). In mouse cell lines, siRNAs and protein-coding inserts were cloned into MGP and MIG vectors (50). The WT IGF2BP3 and deletion mutants (KH and RRM) with a T7 epitope tag were cloned between BglII and XhoI sites in a MIG-based vector (MSCV-T7 tag-Mutant CDS-IRES-GFP; See Supplemental Table 5). For CRISPR-Cas9-mediated targeting, guide RNAs were designed using the Zhang lab website (http://crispr.mit. $\mathrm{edu} /$ ) and cloned into the LentiCRISPR vector (24). RS4;11 cells were spin-infected at $30^{\circ} \mathrm{C}$ for 90 minutes in the presence of polybrene. Cells were selected with $5 \mu \mathrm{g} / \mathrm{ml}$ of puromycin for 7 days and used for cell proliferation and apoptosis assays. The human B-ALL cell lines RS4;11 (MLL-AF4 translocated; ATCC CRL-1873), NALM6 (gift from K. Sakamoto, Stanford University, Stanford, California, USA), 697 (E2A-PBX1translocated; gift from K. Sakamoto), Reh (TEL-AML1-translocated; ATCC CRL-8286), murine pre-B leukemic cell line 7OZ/3 (ATCC TIB158), and HEK 293T cell line (ATCC CRL-11268) were grown in their corresponding media at $37^{\circ} \mathrm{C}$ in a $5 \% \mathrm{CO}_{2}$ incubator. Lentiviruses and retroviruses were generated as previously described $(50,51)$.

$B M$ transplant and competitive repopulation assay. BM was harvested and spin-infected from 8-week-old CD45.2+ donor C57BL/6J female mice as previously described (50). We also transplanted donor mice with T7 epitope-tagged IGF2BP3 and mutant constructs, including deletion mutants lacking RNA-bining domains. Eight-week-old CD45.1 $1^{+}$recipient B6.SJL-Ptprc-Pep3/BoyJ female mice were lethally irradiated and injected with donor BM 6 hours after irradiation. Eight mice were used per group. In some experiments, the normalized engraftment was calculated as the percent engraftment/transduction efficiency. For competitive repopulation experiments, 8-week-old CD45.1 $1^{+}$donor B6.SJL-Ptprc-Pep3/BoyJ female mice and 8-weekold $\mathrm{CD} 45.2^{+}$donor C57BL/6J female mice were harvested for BM. CD 45.2 $2^{+}$BM was infected with viruses overexpressing MIG, HOXA9, or IGF2BP3. CD 45.1 $1^{+}$and CD 45.2 $2^{+}$BM cells were mixed in a ratio of 1:1 and injected into lethally irradiated 8-week-old CD $45.2^{+}$recipient C57BL/6J female mice. A negative control group had 100\% CD45.1 ${ }^{+}$ $\mathrm{BM}$ cells injected into lethally irradiated 8-week-old CD $45.2^{+}$recipient 
C57BL/6J female mice. Mice were bled at 4, 8, 12, 16, and 20 weeks after BM injection for analysis of the peripheral blood. All mice were purchased from the Jackson Laboratory and housed under pathogenfree conditions at UCLA.

Flow cytometry. Blood, BM, thymus, and spleen were collected from the mice under sterile conditions at 27 weeks after transplant. Single cell suspensions were lysed in red blood cell lysis buffer. Fluorochrome-conjugated antibodies were used for staining. The list of antibodies used is provided in Supplemental Table 6. For intracellular staining, after initial staining with surface marker antibodies and fixation with $1 \%$ paraformaldehyde (PFA), cells were incubated with antibodies against intracellular antigens (Ki67, Cdk6, and Myc) in 1\% Triton containing MACS buffer. After 30 minutes of staining at $4^{\circ} \mathrm{C}$, cells were washed twice with PBS and fixed with $1 \%$ PFA. Flow cytometry was performed at the UCLA JCCC and at the Broad Stem Cell Research Flow Core. Analysis was performed using FlowJo software.

Histopathology. Fixation and sectioning has been described previously (51). Analysis was performed by a board certified hematopathologist (D.S. Rao).

iCLIP. iCLIP was performed as previously described (52). Briefly, RS4;11 or REH cells were irradiated with UV-C light to form irreversible covalent cross-link proteins to nucleic acids in vivo. After cell lysis, RNA was partially fragmented using micrococcal nuclease, and IGF2BP3-RNA complexes were immunopurified with anti-IGF2BP3 antibody (MBL International Corporation) immobilized on protein A-coated magnetic beads (Invitrogen). After stringent washing and dephosphorylation (FastAP, Fermentas), RNAs were ligated at their 3' ends to a 3' preadenylated RNA adaptor, radioactively labeled, run using MOPS-based protein gel electrophoresis, and transferred to a nitrocellulose membrane. Protein-RNA complexes 15-80 kDa above free protein were cut from the membrane, and RNA was recovered by proteinase $\mathrm{K}$ digestion under denaturing (3.5 M urea) conditions. The oligonucleotides for reverse transcription contained 2 inversely oriented adaptor regions adapted from the Bioo NEXTflex small RNA library preparation kit (Bioo Scientific), separated by a BamHI restriction site and a barcode region at their $5^{\prime}$ end containing a 4-nt experiment-specific barcode within a 5-nt random barcode to mark individual cDNA molecules. cDNA molecules were size purified using denaturing PAGE, circularized by CircLigase II (Epicenter, Illumina), annealed to an oligonucleotide complementary to the restriction site and cut using BamHI (New England Biolabs Inc.). Linearized cDNAs were then PCR-amplified using Immomix PCR Master Mix (Bioline) with primers (Bioo Scientific) complementary to the adaptor regions and subjected to high throughput sequencing using Illumina HiSeq. A more detailed description of the iCLIP protocol has been published (53). The data discussed in this publication have been deposited in NCBI's GEO (54) (GSE76931).

iCLIP data analysis. Following transcriptomic and genomic alignment with "TopHat2" (55), individual reads were truncated to their 5 ' ends to represent the site of cross-linking. To denote specific sites of protein-RNA interaction, 30-bp regions of enrichment over background were determined using "Piranha" (56) in zero-truncated negative binomial mode with a custom local covariate. The covariate was calculated by uniformly distributing the cross-link number of each 30-bp bin across the neighboring 6 bins ( 3 on each side) to control for regions of overall higher depth as an indication of protein-RNA interaction. Adjacent bins with significant $P$ values from Piranha $(\alpha<0.05)$ were combined into single regions. Only overlapping peak regions found to be statistically significant in all 3 replicates were considered biologically reproducible candidates for further analysis. To derive the intragenic distributions of iCLIP-Seq sites, we queried the UCSC Genome Browser MySQL database for hy19 (57) and determined the nearest overlapping gene based first on CCDS gene annotations (58) to determine the canonical ORF and second on Gencode V19 comprehensive for other features (59). Following this, the nearest intragenic anchor (transcription start site, start codon, $5^{\prime}$ splice site, $3^{\prime}$ splice site, stop codon, and polyadenylation site) was recorded, and the spliced distance to the nearest ORF boundary was calculated. As a background control, uniformly distributed cross-link sites were simulated by pseudo-random intervals from hg19 using "bedtools" (60). The intragenic distribution of these sites was determined following the same methodology as the iCLIP cross-link sites.

To determine the binding specificity of IGF2BP3, a 10-nt window surrounding each cross-link site occurring within a biologically reproducible and statistically significant peak from a last exon was extracted, and the counts of each n-mer (4 through 6) were calculated. Random 20-bp intervals from a window of 100-300 nucleotides adjacent to each cross-link site were included as a normalized frequency of $\mathrm{n}$-mers to represent the background probability (p) of observing each n-mer. For each n-mer size, the probability of observing k occurrences of some $\mathrm{n}$-mer out of $\mathrm{N}$ total observations is binomially distributed $(\mathrm{k} \sim \operatorname{Bin}[\mathrm{p}, \mathrm{N}])$ and Poisson approximated, given sufficiently large values for $\mathrm{N}(>1,000)$ and small values of $P(<0.01)$. Individual n-mers with Poisson-approximated $P$ values significant at a $5 \%$ false discovery rate (61) were then aligned with one another and grouped into motifs using k-medoid clustering for optimal values of $\mathrm{k}$ (using average silhouette width). The frequency of occurrence of each nucleotide was then plotted in a position-specific manner for each motif cluster.

RNA immunoprecipitation. Protein A Dynabeads (Invitrogen) in $100 \mathrm{mM}$ sodium phosphate buffer $(\mathrm{pH}$ 8.1) were treated with either IgG mouse antibody (Jackson ImmunoResearch Laboratories Inc.) or $\alpha$-IGF2BP3 (Santa Cruz Biotechnology Inc.) for 1 hour at $4^{\circ} \mathrm{C}$. Beads were then washed $3 \times$ with RSB-100 buffer (10 mM Tris- $\mathrm{Cl}$ [pH 7.4], $\left.100 \mathrm{mM} \mathrm{NaCl}, 2.5 \mathrm{mM} \mathrm{MgCl}_{2}, 0.5 \% \mathrm{NP}-40\right)$. Cytoplasmic lysates were prepared from suspension RS4;11 cells in RSB-100 containing RNase inhibitors. Lysate supernatants were combined with the bead/ antibody, rotated at $4^{\circ} \mathrm{C}$ overnight, and washed, and a portion was removed for Western blot analysis. Pelleted beads were resuspended in Proteinase K buffer (100 mM Tris- $\mathrm{HCl}$ [pH 7.4], $50 \mathrm{mM} \mathrm{NaCl}, 10$ mM EDTA [pH 8.0]). Samples were treated with RQ DNase I (Promega) and then treated with Proteinase K (Ambion); both were incubated at $37^{\circ} \mathrm{C}$. The RNA was extracted with acid phenol-chloroform and precipitated with sodium acetate, absolute ethanol, and coprecipitate GlycoBlue (Ambion). RNA was recovered by centrifugation, washed, and resuspended in RNase-free water. Quantity and quality were checked with Nanodrop and Total RNA NanoBioanalyzer kit (Agilent Technologies). RNA samples (200 $\mu$ g of each sample; triplicates of both IgG controls and $\alpha$-IGF2BP3 immunoprecipitations) were subjected to reverse transcription using the High-Capacity cDNA Reverse Transcription Kit (Thermo Scientific) and subsequent qPCR using the Lightcycler 480 (Roche Diagnostics).

RNA sequencing experiments. RNA was purified from both cytosolic fractions of IGF2BP3-depleted or control RS4;11 cells using TRIReagent LS (Sigma-Aldrich), converted to double-stranded libraries 
using the NEXTflex Rapid Directional qRNA-Seq Library Prep Kit (Bioo Scientific), and sequenced using Illumina HiSeq 2500 platform. High throughput RNA sequencing data generated by Illumina HighSeq 2500 and corresponded to approximately 30-115 million reads per sample (Supplemental Table 4) was mapped to hg19 build of the human genome (Feb. 2009 GRCh37, NCBI Build 37.1) using Bowtie and TopHat software $(62,63)$. All data collection and parsing was performed with Perl, and statistical analyses with R, version 2.14.1. All external library packages used are available on CPAN or CRAN. Differentially expressed genes were identified using DESeq. The data are accessible through NCBI's GEO (GSE76931).

Luciferase assays. The CDK6 3'UTR is approximately 10-kb long while the MYC $3^{\prime} \mathrm{UTR}$ is approximatley $300 \mathrm{bp}$. MYC $\Delta 3$ and MYC $\Delta 4$ are mutant MYC 3'UTRs lacking IGF2BP3 binding sites determined from iCLIP data. Primers were designed to exclude these binding sites, fusion PCR was completed, and MYC and MYC $\Delta 3^{\prime}$ UTRs were cloned downstream of firefly luciferase in the pmirGlo vector between the SacI and XhoI sites (64). The CDK6 3'UTR was divided into 5 pieces (CDK6 1-5; $2 \mathrm{~kb}$ each) and cloned individually downstream of the firefly luciferase. 293T cells were transfected with the pmirGlo, 3'UTR, or $\Delta 3^{\prime} \mathrm{UTR}$ containing reporter vectors along with the MIG empty vector, MIG-hIGF2BP3 overexpression vector, MIG-T7hIGF2BP3 overexpression vector, or MIG-T7-KH/RRM mutant vectors at a 1:10 ratio $(50: 500 \mathrm{ng})$. Cotransfections were performed with Lipofectamine 2000 (Invitrogen) as per the manufacturer's instructions. Cells were lysed after 24 hours, substrate was added, and luminescence was measured on a GloMax-Multi Jr (Promega). The ratio of firefly to Renilla luciferase activity was calculated for all samples. The hIGF2BP3/MIG or mutant/MIG luminescence for the pmirGlo empty vector was used as a normalization control.

Statistics. Data represent mean \pm SD for continuous numerical data. One-way ANOVA followed by Bonferroni's multiple comparisons test or 2-tailed Student's $t$ tests were performed using GraphPad Prism software and applied to each experiment as described in the figure legends. A $P$ value less than 0.05 was considered significant. ${ }^{*} P<0.05,{ }^{* *} P<0.01,{ }^{* *} P<0.001$, and ${ }^{* * *} P<0.0001$.

Study approval. Written informed consent was obtained from all of the parents of the patients by the Italian Association of Pediatric Hematology and Oncology (AIEOP) and the Berlin-Frankfurt-Muenster (BFM) ALL-2000 trial. The University of Padova IRB approved all procedures, and the study was considered exempt from review at UCLA. Peripheral blood mononuclear cells derived from anonymized donors were obtained from the Center for AIDS Research Virology Core Lab at UCLA or following diagnostic work from the UCLA Department of Pathology and Laboratory Medicine with written consent and IRB approval. All mouse experimental procedures were conducted with the approval of the UCLA Chancellor's Animal Research Committee (ARC).

\section{Author contributions}

JKP designed the study, performed the experiments, analyzed the data, and wrote the paper. JRS and DSR designed the study, analyzed the data, and wrote the paper. TMT and JMH performed the experiments, analyzed the data, and wrote the paper. JRC and TRF performed the experiments. TSW, SK, MT, WY, MP, and GB analyzed the data.

\section{Acknowledgments}

We thank members of the Rao lab for their helpful discussions. We thank Parth Patel, May Paing, Norma Iris Rodriguez-Malave, Jennifer King, Jaspal Bassi, Kim Pioli, Nolan Ung, and Jaime Anguiano for their technical support. This work was supported by NIH grant R01 CA166450, a seed grant from the UCLA JCCC, and a Career Development Award K08CA133521 (to D.S. Rao), as well as by NIH grants R01GM109146 and R21AG042003 (to J.R. Sanford). J.R. Contreras was supported by the Tumor Immunology Training Grant, NIH T32CA009120. T.R. Fernando was supported by Tumor Biology Training Grant NIH T32CA009056. J.M. Howard was supported by R41HG007336. Flow cytometry was performed in the UCLA JCCC/CFAR Flow Cytometry Core Facility that is supported by NIH AI-28697, P30CA016042, the JCCC, the UCLA AIDS Institute, and the David Geffen School of Medicine at UCLA.

Address correspondence to: Dinesh S. Rao, Department of Pathology and Laboratory Medicine, David Geffen School of Medicine at UCLA, 650 Charles E Young Drive, 12-272 Factor, Los Angeles, California 90095, USA. Phone: 310.825.1675; E-mail: drao@mednet.ucla.edu.

Jayanth Kumar Palanichamy's present address is: Department of Biochemistry, All India Institute of Medical Sciences, New Delhi, India.
1. Mullighan CG. Molecular genetics of B-precursor acute lymphoblastic leukemia. J Clin Invest. 2012;122(10):3407-3415.

2. Mullighan CG, Downing JR. Genome-wide profiling of genetic alterations in acute lymphoblastic leukemia: recent insights and future directions. Leukemia. 2009;23(7):1209-1218.

3. Krivtsov AV, Armstrong SA. MLL translocations, histone modifications and leukaemia stem-cell development. Nat Rev Cancer. 2007;7(11):823-833.

4. Krivtsov AV, et al. H3K79 methylation profiles define murine and human MLL-AF4 leukemias. Cancer Cell. 2008;14(5):355-368.

5. Robinson BW, et al. Abundant anti-apoptotic BCL-2 is a molecular target in leukaemias with $\mathrm{t}(4 ; 11)$ translocation. Br J Haematol. 2008;141(6):827-839.

6. Jiang X, et al. Blockade of miR-150 matura- tion by MLL-fusion/MYC/LIN-28 is required for MLL-associated leukemia. Cancer Cell. 2012;22(4):524-535.

7. Placke T, et al. Requirement for CDK6 in MLL-rearranged acute myeloid leukemia. Blood. 2014;124(1):13-23.

8. Jude CD, Climer L, Xu D, Artinger E, Fisher JK, Ernst P. Unique and independent roles for MLL in adult hematopoietic stem cells and progenitors. Cell Stem Cell. 2007;1(3):324-337.

9. Ernst P, Mabon M, Davidson AJ, Zon LI, Korsmeyer SJ. An Mll-dependent Hox program drives hematopoietic progenitor expansion. Curr Biol. 2004;14(22):2063-2069.

10. Rozovskaia T, et al. Upregulation of Meis1 and HoxA9 in acute lymphocytic leukemias with the $\mathrm{t}(4: 11)$ abnormality. Oncogene. 2001;20(7):874-878.
11. Imamura $\mathrm{T}$, et al. Frequent co-expression of HoxA9 and Meis1 genes in infant acute lymphoblastic leukaemia with MLL rearrangement. $\mathrm{Br} \mathrm{J}$ Haematol. 2002;119(1):119-121.

12. Somervaille TC, et al. Hierarchical maintenance of MLL myeloid leukemia stem cells employs a transcriptional program shared with embryonic rather than adult stem cells. Cell Stem Cell. 2009;4(2):129-140.

13. Palanichamy JK, Rao DS. miRNA dysregulation in cancer: towards a mechanistic understanding Front Genet. 2014;5:54.

14. Abdelmohsen K, et al. 7SL RNA represses p53 translation by competing with HuR. Nucleic Acids Res. 2014;42(15):10099-10111.

15. Lederer M, Bley N, Schleifer C, Hüttelmaier S. The role of the oncofetal IGF2 mRNA-binding protein 3 (IGF2BP3) in cancer. Semin Cancer Biol. 
2014;29:3-12.

16. Bell JL, et al. Insulin-like growth factor 2 mRNA-binding proteins (IGF2BPs): post-transcriptional drivers of cancer progression? Cell Mol Life Sci. 2013;70(15):2657-2675.

17. Fadare $\mathrm{O}$, et al. Expression of the oncofetal protein IGF2BP3 in endometrial clear cell carcinoma: assessment of frequency and significance. Hum Pathol. 2013;44(8):1508-1515.

18. Suvasini $\mathrm{R}$, et al. Insulin growth factor-2 binding protein 3 (IGF2BP3) is a glioblastoma-specific marker that activates phosphatidylinositol 3-kinase/mitogen-activated protein kinase (PI3K/MAPK) pathways by modulating IGF-2. JBiol Chem. 2011;286(29):25882-25890.

19. Schaeffer DF, et al. Insulin-like growth factor 2 mRNA binding protein 3 (IGF2BP3) overexpression in pancreatic ductal adenocarcinoma correlates with poor survival. BMC Cancer. 2010;10:59.

20. Kobel M, et al. IGF2BP3 (IMP3) expression is a marker of unfavorable prognosis in ovarian carcinoma of clear cell subtype. Mod Pathol. 2009;22(3):469-475.

21. Fernando TR, et al. LncRNA Expression discriminates karyotype and predicts survival in B-lymphoblastic leukemia. Mol Cancer Res. 2015;13(5):839-851.

22. Tsutsumi S, et al. Two distinct gene expression signatures in pediatric acute lymphoblastic leukemia with MLL rearrangements. Cancer Res. 2003;63(16):4882-4887.

23. Dawson MA, et al. Inhibition of BET recruitment to chromatin as an effective treatment for MLL-fusion leukaemia. Nature. 2011;478(7370):529-533.

24. Shalem O, et al. Genome-scale CRISPR-Cas 9 knockout screening in human cells. Science. 2014;343(6166):84-87.

25. Hardy RR, Shinton SA. Characterization of $B$ lymphopoiesis in mouse bone marrow and spleen. Methods Mol Biol. 2004;271:1-24.

26. Aifantis I, Raetz E, Buonamici S. Molecular pathogenesis of T-cell leukaemia and lymphoma. Nat Rev Immunol. 2008;8(5):380-390.

27. Chen EY, et al. Enrichr: interactive and collaborative HTML5 gene list enrichment analysis tool. BMC Bioinformatics. 2013;14:128.

28. Grimson A, Farh KK, Johnston WK, GarrettEngele P, Lim LP, Bartel DP. MicroRNA targeting specificity in mammals: determinants beyond seed pairing. Mol Cell. 2007;27(1):91-105.

29. Wachter K, Kohn M, Stohr N, Huttelmaier S. Subcellular localization and RNP formation of IGF2BPs (IGF2 mRNA-binding proteins) is modulated by distinct RNA-binding domains. Biol Chem. 2013;394(8):1077-1090.

30. Bernt KM, Armstrong SA. A role for DOT1L in MLL-rearranged leukemias. Epigenomics. 2011;3(6):667-670.

31. Kharas MG, et al. Musashi-2 regulates normal hematopoiesis and promotes aggressive myeloid leukemia. Nat Med.2010;16(8):903-908.

32. Abdelmohsen K, Gorospe M. Posttranscriptional regulation of cancer traits by HuR. Wiley Interdiscip Rev RNA. 2010;1(2):214-229.

33. Guenther MG, et al. Aberrant chromatin at genes encoding stem cell regulators in human mixed-lineage leukemia. Genes Dev. 2008;22(24):3403-3408.

34. Tang $\mathrm{H}$, et al. IMP3 as a supplemental diagnostic marker for Hodgkin lymphoma. Hum Pathol. 2013;44(10):2167-2172.

35. Hartmann EM, et al. Increased tumor cell proliferation in mantle cell lymphoma is associated with elevated insulin-like growth factor 2 mRNA-binding protein 3 expression. Mod Pathol. 2012;25(9):1227-1235.

36. King RL, Pasha T, Roullet MR, Zhang PJ, Bagg A. IMP- 3 is differentially expressed in normal and neoplastic lymphoid tissue. Hum Pathol. 2009;40(12):1699-1705.

37. Liao B, Hu Y, Herrick DJ, Brewer G. The RNA-binding protein IMP-3 is a translational activator of insulin-like growth factor II leader-3 mRNA during proliferation of human K562 leukemia cells. J Biol Chem. 2005;280(18):18517-18524.

38. Stoskus M, Gineikiene E, Valceckiene V, Valatkaite B, Pileckyte R, Griskevicius L. Identification of characteristic IGF2BP expression patterns in distinct B-ALL entities. Blood Cells Mol Dis. 2011;46(4):321-326.

39. Lu D, et al. IMP3, a new biomarker to predict progression of cervical intraepithelial neoplasia into invasive cancer. Am J Surg Pathol. 2011;35(11):1638-1645.

40. Chen CL, et al. Reciprocal regulation by TLR4 and TGF- $\beta$ in tumor-initiating stem-like cells. JClin Invest. 2013;123(7):2832-2849.

41. Wagner $\mathrm{M}$, et al. Transgenic overexpression of the oncofetal RNA binding protein KOC leads to remodeling of the exocrine pancreas. Gastroenterology. 2003;124(7):1901-1914.

42. Jonson L, Christiansen J, Hansen TV, Vikesa J, Yamamoto Y, Nielsen FC. IMP3 RNP safe houses prevent miRNA-directed HMGA2 mRNA decay in cancer and development. Cell Rep. 2014;7(2):539-551.

43. Copley MR, et al. The Lin28b-let-7-Hmga2 axis determines the higher self-renewal potential of fetal haematopoietic stem cells. Nat Cell Biol. 2013;15(8):916-925.

44. Toledano H, D'Alterio C, Czech B, Levine E, Jones DL. The let-7-Imp axis regulates ageing of the Drosophila testis stem-cell niche. Nature. 2012;485(7400):605-610.

45. Yan L, et al. Regulation of tumor cell migration and invasion by the H19/let-7 axis is antagonized by metformin-induced DNA methylation. Oncogene. 2015;34(23):3076-3084.

46. Chen MT, et al. ZFP36L1 promotes monocyte/ macrophage differentiation by repressing CDK6. SciRep. 2015;5:16229.

47. Liu L, et al. Competition between RNA-binding proteins CELF1 and HuR modulates MYC translation and intestinal epithelium renewal. Mol Biol Cell. 2015;26(10):1797-1810.

48. Weidensdorfer D, et al. Control of c-myc mRNA stability by IGF2BP1-associated cytoplasmic RNPs. RNA. 2009;15(1):104-115.

49. Yang F, et al. Long non-coding RNA GHET1 promotes gastric carcinoma cell proliferation by increasing c-Myc mRNA stability. FEBS J. 2014;281(3):802-813.

50. Rao DS, O'Connell RM, Chaudhuri AA, GarciaFlores Y, Geiger TL, Baltimore D. MicroRNA-34a perturbs $B$ lymphocyte development by repressing the forkhead box transcription factor Foxp1. Immunity. 2010;33(1):48-59.

51. O'Connell RM, Balazs AB, Rao DS, Kivork C, Yang L, Baltimore D. Lentiviral vector delivery of human interleukin-7 (hIL-7) to human immune system (HIS) mice expands T lymphocyte populations. PLoS One. 2010;5(8):e12009.

52. Konig J, et al. iCLIP reveals the function of hnRNP particles in splicing at individual nucleotide resolution. Nat Struct Mol Biol. 2010;17(7):909-915.

53. Huppertz I, et al. iCLIP: protein-RNA interactions at nucleotide resolution. Methods. 2014;65(3):274-287.

54. Edgar R, Domrachev M, Lash AE. Gene Expression Omnibus: NCBI gene expression and hybridization array data repository. Nucleic Acids Res. 2002;30(1):207-210.

55. Kim D, Pertea G, Trapnell C, Pimentel H, Kelley R, Salzberg SL. TopHat2: accurate alignment of transcriptomes in the presence of insertions, deletions and gene fusions. Genome Biol. 2013;14(4):R36

56. Uren PJ, et al. Site identification in high-throughput RNA-protein interaction data. Bioinformatics. 2012;28(23):3013-3020.

57. Meyer LR, et al. The UCSC Genome Browser database: extensions and updates 2013. Nucleic Acids Res. 2013;41(Database issue):D64-D69.

58. Pruitt KD, et al. The consensus coding sequence (CCDS) project: Identifying a common protein-coding gene set for the human and mouse genomes. Genome Res. 2009;19(7):1316-1323.

59. Harrow J, et al. GENCODE: the reference human genome annotation for The ENCODE Project. Genome Res. 2012;22(9):1760-1774.

60. Quinlan AR, Hall IM. BEDTools: a flexible suite of utilities for comparing genomic features. Bioinformatics. 2010;26(6):841-842.

61. Klipper-Aurbach Y, et al. Mathematical formulae for the prediction of the residual beta cell function during the first two years of disease in children and adolescents with insulindependent diabetes mellitus. Med Hypotheses. 1995;45(5):486-490.

62. Trapnell C, Pachter L, Salzberg SL. TopHat: discovering splice junctions with RNA-Seq. Bioinformatics. 2009;25(9):1105-1111.

63. Langmead B, Trapnell C, Pop M, Salzberg SL. Ultrafast and memory-efficient alignment of short DNA sequences to the human genome. Genome Biol. 2009;10(3):R25.

64. Contreras JR, et al. MicroRNA-146a modulates B-cell oncogenesis by regulating Egr1. Oncotarget. 2015;6(13):11023-11037. 